\title{
Powermonads and Tensors of Unranked Effects
}

\author{
Sergey Goncharov and Lutz Schröder \\ Research Department Safe and Secure Cognitive Systems \\ German Research Center for Artificial Intelligence (DFKI GmbH), Bremen, Germany \\ Email: Sergey.Goncharov@dfki.de,Lutz.Schroeder@dfki.de
}

\begin{abstract}
In semantics and in programming practice, algebraic concepts such as monads or, essentially equivalently, (large) Lawvere theories are a well-established tool for modelling generic side-effects. An important issue in this context are combination mechanisms for such algebraic effects, which allow for the modular design of programming languages and verification logics. The most basic combination operators are sum and tensor: while the sum of effects is just their noninteracting union, the tensor imposes commutation of effects. However, for effects with unbounded arities, these combinations need not in general exist. Here, we introduce the class of uniform effects, which includes unbounded nondeterminism and continuations, and prove that the tensor does always exist if one of the component effects is uniform, thus in particular improving on previous results on tensoring with continuations. We then treat the case of nondeterminism in more detail, and give an order-theoretic characterization of effects for which tensoring with nondeterminism is conservative, thus enabling nondeterministic arguments such as a generic version of the Fischer-Ladner encoding of control operators.
\end{abstract}

\section{INTRODUCTION}

Both in actual programming languages and in their semantics and meta-theory, one encounters a wide variety of phenomena that can be subsumed under a broadly understood notion of side-effect, such as various forms of state, input/output, resumptions, backtracking, nondeterminism, continuations, and many more. This proliferation of effects motivates the search for generic frameworks that encapsulate the exact nature of side-effects and support abstract formulations of programs (such as Haskell's generic while-loop), semantic principles, and program logics. A fairly well-established abstraction of this kind is the modelling of side-effects as monads, following seminal work by Moggi [1]; this principle is widely used in programming language semantics (e.g. [2]-[5]) and moreover underlies the incorporation of side-effects in the functional programming language Haskell [6]. Besides supporting generic results that can be instantiated to particular effects at little or no cost, monads allow for a clear delineation of the scope of effects [7]. A more recent development is the advancement of Lawvere theories [8] for the generic modelling of effects, thus emphasizing their algebraic nature [9].

Research supported by the DFG (project PLB, LU708/9-1).
One advantage of these approaches is that they provide for a modular semantics of effects. It has been observed that many effects, such as state, exceptions, and continuations, induce so-called monad transformers that can be seen as adding the respective effect to a given set of effects [10], [11]; again, the notion of monad transformer plays a central role in Haskell. More recently, it has been shown that many monad transformers arise from binary combination operators that join effects in a prescribed way. The most important among these constructions are the sum of effects, which corresponds simply to the disjoint union of algebraic theories, and the tensor, which additionally imposes a commutation condition [12], [13]. E.g., the exception monad transformer is summation with the exception monad, and the state monad transformer is tensoring with the state monad [14]. These combination methods are often mixed; e.g. [15] uses both sums and tensors of nondeterminism with other effects. (Previous work on the specific combination of unbounded nondeterminism and probabilistic choice uses a different form of interaction than imposed by the tensor [16], [17].)

One of the problems that arise with sum and tensor in the context of large Lawvere theories, i.e. theories that can be unranked in the sense that their operations have unbounded arities, such as unbounded nondeterminism or continuations, is that for reasons of size, the combined theories need not exist in general; e.g. we show in recent work [18] that tensors of unranked theories with the theory of lists may fail to exist. In the present work, we introduce the notion of uniform theory, and prove that the tensor of two large Lawvere theories always exists if one of them is uniform. The class of uniform theories includes several variants of nondeterminism (e.g. unbounded and countable, but not finite) as well as, somewhat surprisingly, continuations; thus, our existence result improves a previous result stating that the tensor of any ranked theory with continuations always exists [12].

One may read this result as yielding a number of new monad transformers; we are particularly interested in nondeterminism monad transformers, which we dub powermonads. This leads us to a second problem associated specifically with the tensor: since the tensor imposes a complex algebraic interaction between the component effects, it cannot in general be expected to be conservative in the sense that the components embed into the tensor. 
To deal with this issue in the special case of nondeterminism, we focus on bounded theories $L$, which come with a natural approximation ordering. We begin by giving a simplified construction of tensoring with nondeterminism, which is informed by but technically independent of the general existence result (and, e.g., applies also to tensoring finitary theories with finite nondeterminism, although the latter fails to be uniform): morphisms in the tensor of $L$ with nondeterminism are sets of $L$-morphisms modulo rectangular equivalence, a comparatively simple equivalence that forces uniqueness of tupling morphisms. From there, we obtain a more order-theoretic description of the tensor in terms of closed sets of $L$-morphisms, which leads to a simple characterization of theories for which tensoring with nondeterminism is order-theoretically conservative.

The main reason for our interest in tensoring with nondeterminism is that it yields exactly the free extension of a given theory to a completely additive theory, i.e. one that is enriched over complete join semi-lattices; this amounts to having choice operators that distribute over sequential composition on both sides (hence providing a trace-based rather than a bisimulation-based perspective). Thus, whenever a theory $L$ can be conservatively tensored with nondeterminism, one can conduct equational and order-theoretic proofs in it pretending that $L$ is completely additive. E.g., one can use the well-known translation of imperative constructs [19]

$$
\begin{aligned}
& \text { if } b \text { then } p \text { else } q:=b ? ; p+(\neg b) ? ; q \\
& \text { while } b \text { do } p:=(b ? ; p)^{\star} ;(\neg b) ?
\end{aligned}
$$

that we dub the Fischer-Ladner encoding generically, i.e. for any effect satisfying our conservativity conditions. Besides simplifying the reasoning, this uncovers the nondeterministic flavour of imperative branching [20].

The material is organized as follows. We recall basic facts on monads and Lawvere theories in Section II. In Section III, we review tensor products, and proceed directly to the main existence result for tensors with uniform theories. We discuss additive theories in Section IV, and present our results on conservativity of tensoring with nondeterminism in Section V.

\section{LARGE LAWVERE THEORIES AND MONADS}

In a nutshell, the principle of monadic encapsulation of sideeffects originally due to Moggi [1] and subsequently introduced into the functional programming language Haskell as the principal means of dealing with impure features [6] consists in moving the side effect from the function arrow into the result type of a function: a side-effecting function $X \rightarrow Y$ becomes a pure function $X \rightarrow T Y$, where $T Y$ is a type of side-effecting computations over $Y$; the base example is $T Y=S \rightarrow(S \times Y)$ for a fixed set $S$ of states, so that functions $X \rightarrow T Y$ are functions that may read and update a global state (more examples will be given later). Formally, a monad on the category of sets, presented as a Kleisli triple $\mathbb{T}=\left(T, \eta,{ }_{-}^{\star}\right)$, consists of a function $T$ mapping sets $X$ (of values) to sets $T X$ (of computations), a family of functions $\eta_{X}: X \rightarrow T X$, and a map assigning to every function $f: X \rightarrow T Y$ a function $f^{\star}: T X \rightarrow T Y$ that lifts $f$ from $X$ to computations over $X$. These data are subject to the equations $\eta^{\star}=\mathrm{id}, f^{\star} \eta=f,\left(f^{\star} g\right)^{\star}=f^{\star} g^{\star}$, which ensure that the Kleisli category of $\mathbb{T}$, which has sets as objects and maps $X \rightarrow T Y$ as morphisms, is actually a category, with identities $\eta: X \rightarrow T Y$ and composition $f^{\star} g$. On Set, all monads are strong, i.e. equipped with a natural transformation $X \times T Y \rightarrow T(X \times Y)$ satisfying a number of coherence conditions [1].

Monads were originally intended as abstract presentations of algebraic theories, with $T X$ abstracting the free algebra over $X$, i.e. terms over $X$ modulo provable equality. It has been shown that the algebraic view of monads gives rise to computationally natural operations for effects; e.g. the state monad (with state set $S=V^{L}$ for sets $V$ of values and $L$ of locations) can be algebraically presented in terms of operations lookup and update [9]. Categorically, this shift of viewpoint amounts to generating monads from Lawvere theories. To cover unranked theories, we use the notion of large Lawvere theory [21], introduced into the theory of generic effects in [12]. Generally, we denote hom-sets of a category $\mathbf{C}$ in the form $\mathbf{C}(A, B)$.

Definition 1 (Large Lawvere theory). A large Lawvere theory is given by a locally small category $L$ with small products, together with a strict product preserving identityon-objects functor $I:$ Set $^{o p} \rightarrow L$. We call $I$ the indexing functor, and we denote $I f$ by $[f]$ for a map $f$. A morphism of large Lawvere theories $L_{1} \rightarrow L_{2}$ is a functor $L_{1} \rightarrow L_{2}$ that commutes with the indexing functors (and hence preserves small products). A model of a large Lawvere theory $L$ in a category $\mathbf{C}$ with small products is a small product preserving functor $L \rightarrow \mathbf{C}$.

The algebraic intuition behind these definitions is that the objects of a large Lawvere theory are sets $n, m, k, \ldots$ of variables, and morphisms $n \rightarrow m$ are $m$-tuples of terms over $n$, or substitutions from $m$ into terms over $n$. The indexing functor prescribes the effect of rearranging variables in terms. The notion of model recalled above implies that Lawvere theories provide a representation of effects that is independent of the base category $\mathbf{C}$, and given enough structure on $\mathbf{C}$, a Lawvere theory will induce a monad on C. E.g., in categories of domains, the theory of finite non-blocking nondeterminism (Example 2.2 below) induces precisely the Plotkin powerdomain monad (while the Hoare and Smyth powerdomains require enriched Lawvere theories) [22].

It is well-known that large Lawvere theories and strong monads on Set form equivalent (overlarge) categories [12], [21]. The equivalence maps a large Lawvere theory $L$ to the monad $T_{L} X=L(X, 1)$ (we elide the full description), 
and a monad $T$ to the dual of its Kleisli category. We therefore largely drop the distinction between monads and large Lawvere theories, and freely transfer concepts and examples from one setting to the other; occasionally we leave the choice open by just using the term effect.

We say that a large Lawvere theory $L$ is ranked if it can be presented by operations (and equations) of arity less than $\kappa$ for some cardinal $\kappa$; otherwise, $L$ is unranked. Categorically, $L$ having rank $\kappa$ amounts to preservation of $\kappa$-directed colimits by the induced monad. If $L$ has rank $\kappa$, then $L$ is determined by its full subcategory spanned by the sets of cardinality less than $\kappa$. If $L$ has rank $\omega$, we say that $L$ is finitary.

Example 2. 1) Global state: as stated initially, $T X=$ $S \rightarrow(S \times X)$ is a monad (for this and other standard examples, we omit the description of the remaining data), the well-known state monad. A variant is the partial state monad $T X=S \rightarrow(S \times X)_{\perp}$, where $X_{\perp}$ extends $X$ by a fresh element $\perp$ representing non-termination. (This induces a relational model of non-termination in the spirit of PDL and related formalisms; a domain-theoretic treatment of nontermination requires a domain-enriched Lawvere theory in which $\perp$ is explicitly a bottom element).

2) Nondeterminism: the unranked large Lawvere theory $L_{\mathcal{P}}$ for nondeterminism arises from the powerset monad $\mathcal{P}$. It has $m$-tuples of subsets of $n$ as morphisms $n \rightarrow m$. Variants arise on the one hand by restricting to nonempty subsets, thus ruling out non-termination, and on the other hand by bounding the cardinality of subsets. We denote nonemptyness by a superscript *, and cardinality bounds by subscripts. E.g., the large Lawvere theory $L_{\mathcal{P}^{*}}$ describes finite non-blocking nondeterminism; its morphisms $n \rightarrow m$ are $m$-tuples of nonempty finite subsets of $n$. Yet another variant arises by replacing sets with multisets, i.e. maps $X \rightarrow(\mathbb{N} \cup\{\infty\})$, thus modelling weighted nondeterminism [23] as a large Lawvere theory $L_{\text {mult }}$.

3) Continuations: The continuation monad maps a set $X$ to the set $(X \rightarrow R) \rightarrow R$, for a fixed set $R$ of results. The corresponding unranked large Lawvere theory $L_{\text {cont }}^{R}$ has maps $m \rightarrow((n \rightarrow R) \rightarrow R)$ as morphisms $n \rightarrow m$.

4) Input/Output: For a given set $I$ of input symbols, the Lawvere theory $L_{I}$ for input is generated by a single $I$ ary operation in; it is an absolutely free theory, i.e. has no equations. Similarly, given a set $O$ of output symbols, the Lawvere theory $L_{O}$ for output is generated by unary operations out $t_{o}$ for $o \in O$.

Further effects that fit the algebraic framework are exceptions $(T X=X+E)$, resumptions $(R X=\mu Y . T(X+Y)$ for a given base effect $T$ ) and many more.

Notation 3. Let $L$ be a large Lawvere theory. For an object $n$ of $L$ and $i \in n$, we let $\kappa_{i}$ denote the map $1 \rightarrow n$ that picks $i$. Thus, the $\kappa_{i}$ induce product projections $\left[\kappa_{i}\right]: n \rightarrow 1$ in $L$. Given two sets $n$ and $m$, their Set-product $n \times m$ can be viewed as the sum of $m$ copies of $n$ in Set, and hence as the $m$-th power of $n$ in $L$. This induces for every $f: n \rightarrow k$ in $L$ the morphisms $f \otimes m: n \times m \rightarrow k \times m$ and $m \otimes f: m \times n \rightarrow m \times k$.

A convenient way of denoting generic computations is the so-called computational metalanguage [1], which has found its way into functional programming in the shape of Haskell's do-notation. We briefly outline the version of the metalanguage we use below.

The metalanguage serves to denote morphisms in the underlying category of a given monad, using the monadic structure; since large Lawvere theories correspond to monads on Set, the metalanguage just denotes maps in our setting. We let a signature $\Sigma$ consist of a set $\mathcal{B}$ of base types, to be interpreted as sets, and a collection of typed function symbols to be interpreted as functions. Here, we assume that types $A, B \in \mathcal{T}$ are generated from the base types by the grammar

$$
A, B::=1|A \in \mathcal{B}| A+B|A \times B| T A
$$

where + and $\times$ are interpreted as set theoretic sum and product, respectively, 1 is a singleton set, and $T$ is application of the given monad. We then have standard formation rules for terms-in-context $\Gamma \triangleright t: A$, read 'term $t$ has type $A$ in context $\Gamma$, where a context is a list $\Gamma=\left(x_{1}: A_{1}, \ldots, x_{n}: A_{n}\right)$ of typed variables (later, contexts will mostly be omitted):

$$
\begin{gathered}
\frac{x: A \in \Gamma}{\Gamma \triangleright x: A} \quad \frac{f: A \rightarrow B \in \Sigma \quad \Gamma \triangleright t: A}{\Gamma \triangleright f(t): B \quad} \frac{\Gamma \triangleright t: A}{\Gamma \triangleright \star} \\
\frac{\Gamma \triangleright t: A \quad \Gamma \triangleright u: B}{\Gamma \triangleright\langle t, u\rangle: A \times B} \quad \frac{\Gamma \triangleright t: A}{\Gamma \triangleright \text { fst } t: B \quad \Gamma \triangleright \operatorname{snd} t: B} \\
\frac{\Gamma \triangleright s: A+B}{\Gamma \triangleright \operatorname{case} s \text { of inl } x \mapsto t ; \operatorname{inr} y \mapsto u: C} \\
\frac{\Gamma \triangleright t: A}{\Gamma \triangleright \operatorname{inl} t: A+B} \quad \frac{\Gamma \triangleright t: B}{\Gamma \triangleright \operatorname{inr} t: A+B}
\end{gathered}
$$

This syntax supports, e.g., the standard encoding of the ifoperator as

$$
\text { if } b \text { then } p \text { else } q=\text { case } b \text { of inl } \star \mapsto p \text {; inr } \star \mapsto q \text {, }
$$

for $b: 2$, where $2=1+1$. Beyond this, we have monadic term constructors

$$
\frac{\Gamma \triangleright t: A}{\Gamma \triangleright \operatorname{ret} t: T A} \quad \frac{\Gamma \triangleright p: T A \quad \Gamma, x: A \triangleright q: T B}{\Gamma \triangleright \operatorname{do} x \leftarrow p ; q: T B}
$$

called return and binding, respectively. Return is interpreted by the unit $\eta$ of the monad, and can be thought of as returning a value. A binding do $x \leftarrow p$; $q$ executes $p$, binds its result to $x$, and then executes $q$, which may use $x$ (if not, mention of $x$ may be omitted). It is interpreted using Kleisli composition and strength, where the latter serves to 
propagate the context $\Gamma$ [1]. In consequence, one has the monad laws

$$
\begin{gathered}
\text { do } x \leftarrow p ; \text { ret } x=p \quad \text { do } x \leftarrow \operatorname{ret} a ; p=p[a / x] \\
\text { do } x \leftarrow(\text { do } y \leftarrow p ; q) ; r=\text { do } x \leftarrow p ; y \leftarrow q ; r
\end{gathered}
$$

Terms of a type $T A$ are called programs.

\section{TENSORS OF LARGE LAWVERE THEORIES}

One of the key benefits of the monadic modelling of effects is that it allows for a modular treatment, where effects are combined from basic building blocks according to the demands of the programming task at hand. In current programming practice (specifically in Haskell [24]), this is typically achieved by generalizing a given effect to a monad transformer [1], [11], i.e. a function that maps monads to monads, in the process extending them with a given effect. For instance, the state monad transformer $S T$ for a given set $S$ of states maps a given monad $T$ to the monad $S T(T)$ with $S T(T)(X)=S \rightarrow T(S \times X)$. Monad transformers are very general, but do not support a great deal of meta-theoretic results, as no further properties are imposed on them; e.g., they need not be functorial. It has been shown in [13] that many monad transformers arise from a few basic binary operations on Lawvere theories (equivalently on monads). E.g., the exception monad transformer, which maps a monad $T$ to the monad $T\left(Z_{-}+E\right)$ for a fixed set $E$ of exceptions, is just summation with $\_+E$; expressed in terms of large Lawvere theories, the sum $L_{1}+L_{2}$ of two effects $L_{1}, L_{2}$ is simply the disjoint union of the associated theories, i.e. is universal w.r.t. having morphisms $L_{1} \rightarrow L_{1}+L_{2} \leftarrow L_{2}$. Another important operation is the tensor which additionally imposes a strong form of interaction between the component theories in the form of a commutation law.

Definition 4 (Tensor). [12] The tensor $L_{1} \otimes L_{2}$ of large Lawvere theories $L_{1}, L_{2}$ is the large Lawvere theory which is universal w.r.t. having commuting morphisms $L_{1} \rightarrow L_{1} \otimes$ $L_{2} \leftarrow L_{2}$ (elided in the notation), if such a universal theory exists. Here, commutation is satisfaction of the tensor law, i.e. given $f_{1}: n_{1} \rightarrow m_{1}$ in $L_{1}$ and $f_{2}: n_{2} \rightarrow m_{2}$ in $L_{2}$ we demand commutativity of the diagram

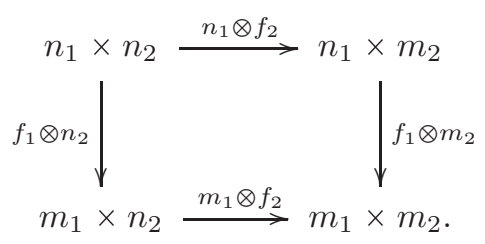

By the equivalence between large Lawvere theories and monads, this induces also a notion of tensor of monads [12]. The computational meaning of the commutation condition becomes clearer in the computational metalanguage: if we extended the metalanguage with subtypes $T_{i} A$ of $T A$ interpreted using the component monads $T_{1}, T_{2}$ of the tensor
$T=T_{1} \otimes T_{2}$, it amounts to the equality

$$
\begin{aligned}
\text { do } x_{1} \leftarrow p_{1} ; x_{2} \leftarrow p_{2} ; \operatorname{ret}\left\langle x_{1}, x_{2}\right\rangle= & \\
& \quad \text { do } x_{2} \leftarrow p_{2} ; x_{1} \leftarrow p_{1} ; \operatorname{ret}\left\langle x_{1}, x_{2}\right\rangle
\end{aligned}
$$

in context $\Gamma_{1}, \Gamma_{2}$, where $\Gamma_{i} \triangleright p_{i}: T_{i} A_{i}$ for $i=1,2$; i.e. programs having only effects from $T_{1}$ do not interfere with programs having only effects from $T_{2}$.

Example 5. [14] Tensoring with the state monad $T X=$ $S \rightarrow(S \times X)$ yields exactly the standard state monad transformer (in particular, tensors with $T$ always exist).

Sum and tensor of large Lawvere theories need not exist in general. This is a size issue - if arities of operations are unbounded, then the terms over a given set of variables need not form a set. E.g., the sum $L_{1}+L_{2}$ of almost any unranked large Lawvere theory $L_{1}$ and the theory $L_{2}$ generated by a single unary operation and no equations fails to exist [25]. Generally, the tensor has a better chance to exist than the sum, since it introduces additional equations, and in fact existence of the sum implies existence of the tensor [12]. Nevertheless, the tensor of two large Lawvere theories may fail to exist even when one of the component theories is ranked (of course, it does exist in case both components are ranked); e.g. there are theories whose tensor with the list theory fails to exist [18]. We proceed to show that the tensor exists whenever one of the component theories is uniform in the sense defined presently.

Definition 6 (Uniformity). Let $L$ be a large Lawvere theory. The constants of $L$ are the elements of $c_{L}:=L(0,1)$. For every set $n$ we denote by $c_{L}^{n}: n \rightarrow n+c_{L}$ the morphism [id] $\times \prod_{f \in c_{L}} f$. We say that $L$ is uniform if for every $L$ morphism $f: n \rightarrow m$ there exists a generic morphism, i.e. a morphism $\hat{f}: k \rightarrow 1$ for some set $k$ such that there exists a set-function $\mathrm{u}: k \times m \rightarrow n+c_{L}$ with $f=(\hat{f} \otimes m)[\mathrm{u}] \mathrm{c}_{L}^{n}$.

In other words, a theory is uniform if all terms over a given set $n$ of variables can be obtained from a single generic term $\hat{f}$, possibly having more variables, by substituting for the variables of $\hat{f}$ either variables from $n$ or constants. The relevance to existence of tensors is clear: if a theory $L_{2}$ is uniform, then the tensor law of a putative tensor $L_{1} \otimes L_{2}$ can always be made to apply to a term that has, say, a top layer of operations from $L_{1}$ whose arguments have a top layer from $L_{2}$.

Remark 7. It is easy to see that in Definition $6, k$ can be bounded by $\left(n+c_{L}\right)^{m}$.

Example 8. 1) The theory $L_{\mathcal{P}^{*}}$ of non-blocking unbounded nondeterminism is uniform: Recall that a morphism $f: n \rightarrow m$ in $L_{\mathcal{P}^{*}}$ is a family of $m$ nonempty subsets of $n$. As a generic morphism $\hat{f}$ for $f$, we can thus take the full set $n$, seen as a morphism $n \rightarrow 1$, from which any other subset of $n$ can be obtained by identifying some of the variables. 
2) The theory $L_{\mathcal{P}}$ of unbounded nondeterminism is uniform: The argument is analogous as for $L_{\mathcal{P}^{*}}$, except that we now need to use also the constant $\varnothing$ in substitutions in order to obtain the empty set as a substitution instance of the generic morphism $\hat{f}$.

3) The theory $L_{\mathcal{P}_{\omega}}$ of finite nondeterminism fails to be uniform: if $\sup _{i \in m}\left|A_{i}\right|=\infty$ for an infinite family $\left(A_{i}\right)_{i \in m}$ of finite subsets of an infinite set $n$, then there is no single finite set from which all sets $A_{i}$ can be obtained by substituting $\varnothing$ or variables from $n$.

4) The theory $L_{\mathcal{P}_{\omega_{1}}}$ of countable nondeterminism is uniform: any infinite countable subset of $n$ will serve as a generic morphism $\hat{f}$ for any morphism $f: n \rightarrow m$, i.e. any family of at $m$ most countable subsets of $n$.

5) The theory $L_{\text {mult }}$ of unbounded weighted nondeterminism is uniform: Recall from Example 2.2 that a morphism $f: n \rightarrow m$ in $L_{\text {mult }}$ is a family of $m$ multisets over $n$ (i.e. maps $n \rightarrow \mathbb{N} \cup\{\infty\}$ ). As a generic morphism $\hat{f}$, we can take the multiset over $\mathbb{N} \times n$ that contains every element with multiplicity 1 .

Moreover, uniformity also subsumes continuations, a fact that we state and prove separately:

Lemma 9. For every $R$, the continuation theory $L_{\text {cont }}^{R}$ (Example 2.3) is uniform.

Proof: W.1.o.g. $|R| \geqslant 2$. We identify the set of constants of $L_{\text {cont }}^{R}$ with $R$. Let $f: n \rightarrow m$ in $L_{\text {cont }}^{R}$; recall that $L_{\text {cont }}^{R}$ is the dual of the Kleisli category of the continuations monad, i.e. $f$ is a map $m \rightarrow((n \rightarrow R) \rightarrow R)$. Pick $J$ such that $|m| \leqslant\left|R^{J}\right|$; we can assume w.l.o.g. that $m=R^{J}$, as we can just pad out $f: n \rightarrow m$, thought of as a family of $m$ morphisms $n \rightarrow 1$, with sufficiently many copies of one of these morphisms. The required generic morphism for $f$ is $\hat{f}: n+J \rightarrow 1$, defined by

$$
\hat{f}(c)=f(\lambda j \cdot c(\operatorname{inr} j))(\lambda a \cdot c(\operatorname{inl} a))
$$

for $c: n+J \rightarrow R:$ Let $\mathrm{u}:(n+J) \times m \rightarrow n+R$,

$$
\mathrm{u}(x, i)=\text { case } x \text { of inl } y \mapsto \operatorname{inl} y \text {; inr } j \mapsto \operatorname{inr} i(j) \text {. }
$$

Then for $i \in m=R^{J}$ and $k: n \rightarrow R$,

$$
\begin{aligned}
((\hat{f} & \left.\otimes m)[\mathrm{u}] \mathrm{c}_{L}^{n}\right)(i)(k) \\
& =\hat{f}(\lambda x . \text { case } u(x, i) \text { of inl } y \mapsto k(y) ; \operatorname{inr} r \mapsto r) \\
& =\hat{f}(\lambda x . \text { case } x \text { of inl } y \mapsto k(y) ; \operatorname{inr} j \mapsto i(j)) \\
& =f(i)(k) .
\end{aligned}
$$

The main existence result for tensors is as follows.

Theorem 10. Let $L_{1}, L_{2}$ be large Lawvere theories, and let $L_{2}$ be uniform. Then the tensor product $L_{1} \otimes L_{2}$ exists.

Proof sketch: By explicit syntactic construction of the tensor product $L=L_{1} \otimes L_{2}$. One constructs a precursor $\mathbf{C}$ of the tensor whose morphisms $n \rightarrow m$ are equivalence classes of paths $n \rightarrow m$; a single step $k \rightarrow l$ in a path is of the form $f * g$ where $f: p \rightarrow l$ in $L_{2}$ and $g: k \rightarrow p$ in $L_{1}$. The equivalence is the congruence $\sim$ on paths $\left(f_{1} * g_{1} \mid\right.$ $\left.\ldots \mid f_{u} * g_{u}\right)$ generated by $([\mathrm{id}] *[\mathrm{id}]) \sim(D),(f[e] * g) \sim$ $(f *[e] g)$, and

$$
\left.\left(f *\left(n^{\prime} \otimes g\right) \mid\left(f^{\prime} \otimes m\right) * g^{\prime}\right) \sim 0 f\left(f^{\prime} \otimes m^{\prime}\right) *(n \otimes g) g^{\prime}\right) .
$$

Using uniformity of $L_{2}$, one shows that every morphism of $\mathrm{C}$ has a representative of the form

$$
\left(f * g \mid \mathrm{c}_{L_{2}}^{n} *[\mathrm{id}]\right)
$$

(recall notation from Definition 6). One shows moreover that in (1), the domain of $f$ can be taken to be $k=L_{1}(n+$ $c_{L_{2}}, 1$ ), so that $\mathbf{C}$ is locally small. One defines a functor $I: \mathbf{S e t}^{o p} \rightarrow \mathbf{C}$ by $I e=[e] *[\mathrm{id}]$. It turns out that $I$ maps products to weak products, i.e. factorizations through the product exist but need not be unique; this is amended by further quotienting.

Corollary 11. For a large Lawvere theory $L_{1}$, the tensor $L_{1} \otimes L_{2}$ exists if $L_{2}$ is one of the following theories:

- unbounded or countable nondeterminism $L_{\mathcal{P}}, L_{\mathcal{P}_{\omega_{1}}}$;

- unbounded or countable non-blocking nondeterminism $L_{\mathcal{P}^{*}}, L_{\mathcal{P}_{\omega_{1}}^{*}}$

- weighted nondeterminism $L_{\text {mult }}$; or

- continuations $L_{\text {cont }}^{R}$.

Of course, a corresponding result holds for monads. This result induces new monad transformers for nondeterminism, continuations, etc. The existence result for tensoring with continuations improves over previous results stating that the tensor of continuations with any ranked theory exists [12]. The results involving nondeterminism are, to our knowledge, entirely new. We refer to tensoring with any form of nondeterminism as a powermonad construction.

\section{Completely Additive Monads AND THE FISCHER-LADNER ENCODING}

Having shown that the tensor of any effect with nondeterminism always exists, we proceed to show that this amounts to a universal construction of an additive theory, i.e. a theory that includes nondeterministic choice operators which distribute over sequential composition. There are two versions of this phenomenon, with and without blocking (i.e. the empty set); for economy of presentation we concentrate on the case with blocking. We start out with a few notions concerned with blocking.

Definition 12 (Bounded theory). We call a large Lawvere theory $L$ bounded if $|L(0,1)|=1$.

(The term bounded is motivated by the fact that $\perp$ is the bottom of a natural approximation ordering introduced later.) Boundedness is connected to tensoring, as follows. 
Definition 13. We denote by $L_{\perp}$ the large Lawvere theory generated by a constant $\perp$ and no equations.

Lemma 14. A large Lawvere theory $L$ is bounded iff $L \otimes$ $L_{\perp} \cong L$.

We denote the only constant of a bounded theory by $\perp_{0,1}$, and put $\perp_{n, m}=\left(\perp_{0,1} \otimes m\right)[\sharp]: n \rightarrow m$ for all $n, m$, where $\sharp$ is the unique map $0 \rightarrow n$. In the sequel, we mostly write $\perp$ in place of $\perp_{n, m}$. As usual, we have a corresponding notion of bounded monad.

Example 15 (Bounded effects). Besides $L_{\perp}$, basic examples of bounded theories include all forms of nondeterminism with blocking. Similarly, the list monad is bounded ( $\perp$ is the empty list). By Lemma 14, the state monad transformer, being defined by tensoring, preserves boundedness; e.g. the partial state monad $S \rightarrow\left(S \times \_\right) \perp$ and the non-deterministic state monad $S \rightarrow \mathcal{P}\left(S \times{ }_{-}\right)$are bounded.

Definition 16 (Additive theories). [26] A large Lawvere theory $L$ is finitely additive if $L$ is enriched over join semilattices, and completely additive if $L$ is enriched over complete join semilattices (with $\perp$ ).

Again, corresponding notions for monads are implied. Joins serve to model nondeterministic choice. Recall that enrichment amounts to hom-sets carrying the structure in question, and composition preserving it in both arguments; i.e. composition distributes over choice and deadlock on both sides. The enrichment is a property rather than part of the data constituting a (completely or finitely) additive Lawvere theory, as by Lemma 24 proved later, the ordering is uniquely determined by the algebraic structure. The relation of additive theories to tensors is the following.

Lemma 17. For a large Lawvere theory $L$, the following are equivalent.

i) $L$ is completely additive.

ii) $L \cong L \otimes L_{\mathcal{P}}$.

iii) $L$ is bounded and has a family of morphisms $\mathrm{U}_{n}: n \rightarrow$ 1 , where $n$ ranges over all sets, such that for any surjection $\sigma: m \rightarrow n$,

$$
\mathrm{U}_{n}=\mathrm{U}_{m}[\sigma]
$$

and for every L-morphism $f: m \rightarrow 1$,

$$
f\left(\mathrm{U}_{n} \otimes m\right)=\mathrm{U}_{n}(n \otimes f) .
$$

The same equivalence holds for finite additivity, tensoring with $L_{\mathcal{P}_{\omega}}$, and (iii) for finite $n, m$.

The operations $\mathrm{U}_{n}$ are $n$-fold joins, with 0 -fold join $\mathrm{U}_{0}$ necessarily being $\perp$. In other words, a completely additive theory $L$ is one that has nondeterministic choice operators that commute over all operations of $L$ as prescribed by the tensor law. From the above, it is immediate that tensoring a large Lawvere theory $L$ with $L_{\mathcal{P}}$ yields the free completely additive theory over $L$,

i.e. the (overlarge) category of completely additive theories is reflexive in the category of large Lawvere theories.

Example 18. The generic example of a completely additive monad is $\mathcal{P}$, with joins being set unions. More generally, nondeterministic global state, $S \rightarrow \mathcal{P}\left(S \times \_\right)$, is completely additive. A non-example is non-determinism with exceptions, $\mathcal{P}\left(Z_{-}+E\right)$, which has several constants and hence fails to be bounded.

Remark 19. Although completely additive theories are enriched, they can be treated as standard large Lawvere theories - as made explicit in Lemma 17, the completely additive structure is algebraic (although unranked), and hence respected by all product-preserving functors.

We proceed to formalize the example application from the introduction, i.e. to show that completely additive monads indeed allow for a generic Fischer-Ladner encoding of control structures. We base this formalization on the fact that every completely additive monad is a Kleene monad [26], i.e. supports Kleene iteration. Specifically, we can extend the computational metalanguage with operators $\perp$ (deadlock), + (binary choice, interpreted by binary joins), and a generic loop construct

$$
\frac{\Gamma \triangleright p: T A \Gamma, x: A \triangleright q: T A}{\Gamma \triangleright \text { init } x \leftarrow p \text { in } q^{\star}: T A} .
$$

The latter is interpreted as the join of all finite iterations of $q$, prefixed with $x \leftarrow p$ and with the result $x$ of the computation fed through the loop; i.e. init $x \leftarrow p$ in $q^{\star}$ is the join of $p$ and all programs do $x \leftarrow p ; x \leftarrow q ; \ldots ; x \leftarrow q ; q$ where $x \leftarrow q$ appears $n \geqslant 0$ times. Moreover, we include in the signature the test operator ?: $2 \rightarrow T 1$, which sends inr $\star$ to ret $\star$ and inl $\star$ to $\perp$. Of course,,$+ \perp$, and ? are supported already by finitely additive monads. From + , we can define an ordering $\leqslant$ in the usual way via $p \leqslant q \Longleftrightarrow p+q=q$. Issues in the axiomatization of a language with choice and iteration are studied in [26], [27]. Relevant axioms and rules for choice, deadlock, and iteration, including in particular two induction rules for iteration, are shown in Figure 1.

Recall that we have given a definition of the if-operator in terms of the case operator in the base language (Section II), with $2=1+1$ representing the Booleans. The proof of the following result appeals only to right distributivity of sequencing over choice and deadlock; we state it in less generality here only for the sake of brevity.

Proposition 20 (Generic Fischer-Ladner encoding).

1) Given a finitely additive monad $T$, for all $\Gamma \triangleright b: 2$, $\Gamma \triangleright p: T A$ and $\Gamma \triangleright q: T A$,

$$
\text { if } b \text { then } p \text { else } q=\text { do } b ? ; p+\text { do }(\neg b) \text { ?; } q \text {. }
$$




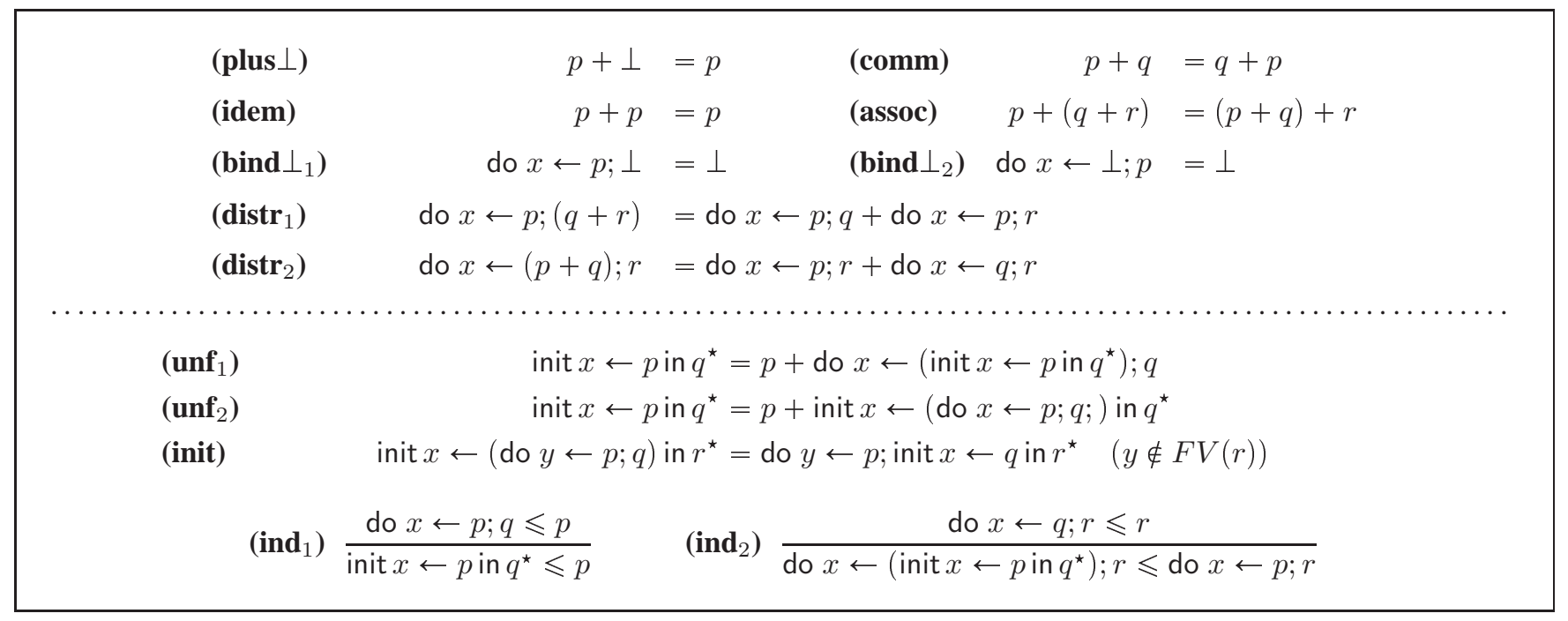

Figure 1. Axioms and rules for nondeterminism (top) and Kleene iteration (bottom)

2) Given a Kleene monad $T$, for every $\Gamma, x: A \triangleright b: 2$ and $\Gamma, x: A \triangleright p: T A$ the map sending $\Gamma, x: A \triangleright q$ to

$$
\Gamma, x: A \triangleright \text { if } b \text { then do } x \leftarrow p ; q \text { else ret } x
$$

has a least fixed point given as the term

$$
\text { do } x \leftarrow\left(\text { init } x \leftarrow \operatorname{ret} x \text { in }(\text { do } b ? ; p)^{\star}\right) ;(\neg b) \text { ?; ret } x \text {. }
$$

The least fixed point in 2) is essentially a while loop, which could be denoted in the form while $b$ do $x \leftarrow p$. Here, the results of $p$ are fed through the loop via the variable $x$; note that the condition $b$ itself does not read the state (being of type 2 instead of T2), but is updated in every iteration of the loop via its dependence on the loop variable $x$.

Remark 21. When absolutely free theories are used as a model of $\mathrm{I} / \mathrm{O}$, preservation of deadlock by sequencing from the left, as imposed by tensoring with $\mathcal{P}$, is hard to justify computationally. A satisfactory way of combining I/O with non-determinism and blocking will require fine-tuned mechanisms between sum and tensor yet to be developed.

\section{Conservativity}

As indicated above, the algebraic complexity of the tensor implies that it is, in general, not at all clear that the component theories map faithfully into the tensor, i.e. that adding a new effect is conservative. In the following we investigate this issue for the powermonad obtained by tensoring with $\mathcal{P}$ (nondeterminism with blocking); in the terminology of the previous section, this amounts to asking for which monads $T$ it can be soundly assumed that they are completely additive, thus enabling, e.g., arguments using the FischerLadner encoding.

Since $L_{\mathcal{P}}$ has a constant $(\varnothing)$, an evident necessary condition for $L \rightarrow L \otimes L_{\mathcal{P}}$ to be faithful is that $L$ can conservatively be made bounded, i.e. $L \rightarrow L \otimes L_{\perp}$ (Definition 13) must be faithful - this is equivalent to $L$ being already bounded if $L$ has a constant, and a complex issue not in scope of the current investigation otherwise (one sufficient condition is that $L$ is generated by equations having the same free variables on both sides). For the sake of readability, we thus restrict the further development to bounded large Lawvere theories.

However, constants are not the only problem: Even tensoring with nonempty powerset $\mathcal{P}^{*}$ can be non-conservative, one counterexample being $\left(\mathcal{P}^{*}\right)^{2} \otimes \mathcal{P}^{*}=\mathcal{P}^{*}$ where $\left(\mathcal{P}^{*}\right)^{2}=$ $\mathcal{P}^{*} \mathcal{P}^{*}$ is the double nonempty powerset monad (which may be thought of as generated by unbounded conjunction and disjunction operators and a distributive law). Collapse of $\left(\mathcal{P}^{*}\right)^{2} \otimes \mathcal{P}^{*}$ to $\mathcal{P}^{*}$ is due to a variant of the well-known Eckmann-Hilton argument [28].

We proceed to give an exact characterization of those theories $L$ for which tensoring with nondeterminism $L_{\mathcal{P}}$ is order-theoretically conservative in a sense to be made precise presently. The main point here is that bounded large Lawvere theories carry a natural preordering:

Definition 22 (Approximation). Let $L$ be a bounded large Lawvere theory. We compare elements of hom-sets $L(n, m)$ under the approximation preorder $\sqsubseteq$, which is the smallest preorder (strictly speaking: family of preorders on hom-sets) with $\perp$ as a bottom element and closed under the rule

$$
\left(\pi_{\sqsubseteq}\right) \frac{\forall i .\left[\kappa_{i}\right] f \sqsubseteq\left[\kappa_{i}\right] g}{h f \sqsubseteq h g}
$$

(equivalently, tupling and composition are monotone).

Roughly, $f \sqsubseteq g$ if $f$ is obtained from $g$ by repeatedly deleting subterms and applying the given equations. It is precisely the approximation preorder which provides the essential handle for characterizing conservativity. The approximation preorder does rely essentially on boundedness, i.e. on the fact that there is exactly one constant. To find a corresponding result for tensoring with non-empty 
non-determinism $\left(\mathcal{P}^{\star}\right)$, i.e. to find a replacement for the approximation preorder in the absence of $\perp$, remains an open problem.

Example 23. 1) The approximation ordering on $L_{\mathcal{P}}$ and its variants is the subset relation. More generally, the approximation ordering coincides with the induced ordering in any additive theory, see Lemma 24 below.

2) In the list monad, $l \sqsubseteq k$ for lists $l, k$ iff $l$ can be obtained from $k$ by deleting some of its entries.

3) The approximation ordering on the theory $L_{\text {mult }}$ of weighted nondeterminism is multiset containment.

4) The approximation ordering on the partial state monad $S \rightarrow\left(S \times{ }_{-}\right)_{\perp}$ is the extension ordering.

Lemma 24. Let $L$ be a finitely additive (hence bounded) large Lawvere theory. Then the approximation preorder $\sqsubseteq$ on $L$ coincides with the order $\leqslant$ induced by the additive structure.

Lemma 25. Every morphism of bounded large Lawvere theories preserves the approximation preorder.

The previous lemma applies in particular to the tensor map $L \rightarrow L \otimes L_{\mathcal{P}}$ for a bounded large Lawvere theory $L$. All this indicates that the relevant notion of conservativity should take into account the approximation preorder.

Definition 26. Let $L$ be a bounded large Lawvere theory. We say that $L$ admits unbounded nondeterminism if the tensor injection $\sigma_{1}: L \rightarrow L \otimes L_{\mathcal{P}}$ is an order embedding, i.e. $\sigma_{1}$ is faithful and reflects the approximation ordering in the sense that $f \sqsubseteq g$ whenever $\sigma_{1}(f) \leqslant \sigma_{1}(g)$.

That is, $L$ admits unbounded nondeterminism if tensoring $L$ with $L_{\mathcal{P}}$ is order-theoretically conservative.

For the remainder of this section, let $L$ be a bounded large Lawvere theory. In a first step, we apply two key simplifications to the description of $L \otimes L_{\mathcal{P}}$ given by the representation according to (1) (Section III): (1) and the subsequent reduction imply that we can represent a morphism $n \rightarrow m$ in $L \otimes L_{\mathcal{P}}$ as an $m$-tuple of sets of $L$-morphisms $n+\{\varnothing\} \rightarrow 1$ (which may be thought of as $L$-terms over $n+\{\varnothing\})$. We can, however

i) represent tuples of sets by sets of tuples using Cartesian products of sets, and

ii) get rid of occurrences of $\varnothing$ in the bottom layer by replacing them with $\perp$, as $\perp=\varnothing$ in the tensor.

Based on these observations, we arrive at a construction of the tensor $L \otimes L_{\mathcal{P}}$ that can be proved correct independently of Theorem 10. To begin, we define a precursor of $L \otimes L_{\mathcal{P}}$, a category $T_{0}$ whose objects are sets and whose morphisms $n \rightarrow m$ are subsets of $L(n, m)$, with composition being complex multiplication $A B=\{f g \mid f \in A, g \in B\}$, and identities $\{$ id $\}$. We have identity-on-objects functors $\sigma_{1}^{0}$ : $L \rightarrow T_{0}$ and $\sigma_{2}^{0}: L_{\mathcal{P}} \rightarrow T_{0}$ defined by $\sigma_{1}^{0} f=\{f\}$, and by $\sigma_{2}^{0}\left(A_{i}\right)=\left\{[e] \mid e: m \rightarrow n, e(i) \in A_{i}\right.$ for all $\left.i\right\}$ for a morphism $\left(A_{i}\right): n \rightarrow m$ in $L_{\mathcal{P}}$, i.e. a family of $m$ subsets $A_{i} \subseteq n$. The category $T_{0}$ inherits a functor $I^{0}: \operatorname{Set}^{o p} \rightarrow T_{0}$ from $L$ via $\sigma_{1}^{0}$; it is easy to see that under the axiom of choice (!), $I^{0}$ maps products to weak products.

We then define a relation $\approx$ on the sets $T_{0}(n, m)$ (strictly speaking: a family of relations on hom-sets) inductively as the smallest equivalence that contains all instances of the axiom scheme

$$
(\perp) \quad\left\{\perp_{n, m}\right\} \approx \varnothing \subseteq L(n, m)
$$

and moreover forces uniqueness of tupling morphisms, i.e. is closed under the infinitary rule

$$
\text { ( } \pi) \frac{\forall i \cdot\left[\kappa_{i}\right] A \approx\left[\kappa_{i}\right] B}{C A \approx C B}
$$

where $L$-morphisms (such as $\left[\kappa_{i}\right]$ ) are meant to convert to singletons when appropriate. We refer to $\approx$ as rectangular equivalence. Implied properties of $\approx$ are symmetry and congruence, the latter holding in particular for tupling and set union. We put $T=T_{0} / \approx$, and obtain functors $I: \operatorname{Set}^{o p} \rightarrow T, \sigma_{1}: L \rightarrow T, \sigma_{2}: L_{\mathcal{P}} \rightarrow T$ by prolongation along $T_{0} \rightarrow T$.

Theorem 27. The category $T$ of sets of L-morphisms modulo rectangular equivalence as constructed above is the tensor product $L \otimes L_{\mathcal{P}}$ of the bounded theory $L$ with unbounded nondeterminism $L_{\mathcal{P}}$.

Similar results hold for tensoring with $L_{\mathcal{P}^{*}}$ (in fact, the construction for $L_{\mathcal{P}^{*}}$ is slightly simpler) and for tensoring finitary theories with $L_{\mathcal{P}_{\omega}}$ or $L_{\mathcal{P}^{*}}$. Salient points in the proof are that the tensor law holds in $T_{0}$ up to rectangular equivalence, and moreover that the general tensor law justifies pointwise composition.

In $L$, we have morphisms $\Delta_{i}=\prod_{j} \delta_{i j}: n \rightarrow n$, where for $i, j \in n, \delta_{i j}: 1 \rightarrow 1$ equals [id] if $i=j$ and $\perp$ otherwise.

Lemma 28. For $f: n \rightarrow m, g: m \rightarrow k$ in $L, f g \approx\left\{f \Delta_{i} g \mid\right.$ $i \in m\}$.

Since the right hand side of the above equivalence is a join in the tensor $L \otimes L_{\mathcal{P}}$, order-theoretic conservativity will imply that it is a join already in $L$. We proceed to develop a characterization of the tensor in terms of order-theoretic closures from this observation.

Definition 29. We say that $A \subseteq L(n, m)$ is closed if $A$ is downclosed and closed under the rule

$$
\text { ( } \Delta) \frac{\forall i . g \Delta_{i} h \in A}{g h \in A} \text {. }
$$

We denote the smallest closed set containing $A \subseteq L(n, m)$ by $\mathrm{cl}(A)$. We write $\mathrm{cl}(f)$ for $\mathrm{cl}(\{f\})$.

The closure $\mathrm{cl}$ completely characterizes equality in the tensor: 
Lemma 30. For $A, B \subseteq L(n, m), A \approx B$ iff $\mathrm{cl}(A)=\mathrm{cl}(B)$. To prove this core fact, we need a preliminary lemma:

Lemma 31. Let $A: n \rightarrow m$ in $T_{0}$. Then for all $a: n \rightarrow m$ and all $b: m \rightarrow k$,

$$
a \in \mathrm{cl}(A) \Longrightarrow b a \in \mathrm{cl}(b A) .
$$

Proof: Show that the set $\{a \in \operatorname{cl}(A) \mid b a \in \operatorname{cl}(b A)\}$ contains $A$, is downward closed, and is closed under $(\Delta)$.

Proof of Lemma 30: Only if: Show that the equivalence $\simeq$ defined by $A \simeq B$ iff $\operatorname{cl}(A)=\operatorname{cl}(B)$ is closed under $(\pi)$ and contains all instances of $(\perp)$. Here, left congruence can conveniently be split off from $(\pi)$ as a separate rule, and closedness under left congruence is proved using Lemma 41.

If: It suffices to show that for $A: n \rightarrow m$ in $T_{0}, A \approx$ $\mathrm{cl}(A)$. Since $\approx$ is congruent w.r.t. set union, it suffices to show that $A \approx A \cup\{f\}$ for all $f \in \operatorname{cl}(A)$, which will follow if we show that the set

$$
\bar{A}:=\{f: n \rightarrow m \mid A \approx A \cup\{f\}\}
$$

(which clearly contains $A$ ) is downward closed and closed under $\Delta$.

- $\bar{A}$ is downward closed: define a preorder $\unlhd$ by $f \unlhd g$ : $\Longleftrightarrow\{f, g\} \approx g$; then $\unlhd$ is easily seen to be closed under $\pi_{\sqsubseteq}$, and hence contains $\sqsubseteq$. Now let $g \in \bar{A}, f \sqsubseteq g$. Then $f \unlhd g$ and therefore

$$
A \cup\{f\} \approx A \cup\{g\} \cup\{f\} \approx A \cup\{g\} \approx A,
$$

using congruence of $\approx$ w.r.t. union.

- $\bar{A}$ is closed under $(\Delta)$ : Let $f: n \rightarrow m, g: m \rightarrow k$, and let $f \Delta_{i} g \in \bar{A}$ for all $i \in m$. Then

$$
A \cup\{f g\} \approx A \cup\left\{f \Delta_{i} g \mid i \in m\right\} \approx A,
$$

using congruence w.r.t. union and Lemma 28.

Consequently, the tensor $L \otimes L_{\mathcal{P}}$ can be regarded as having closed subsets of $L(n, m)$ as morphisms $n \rightarrow m$. The following is, then, more or less immediate.

Theorem 32 (Order-theoretic conservativity). Let $L$ be a bounded large Lawvere theory with approximation preorder $\sqsubseteq$ as defined above, and let $\sigma_{1}: L \rightarrow L \otimes L_{\mathcal{P}}$ be the tensor injection into the powermonad.

1) The following are equivalent:

(i) $\sigma_{1}$ reflects the approximation ordering.

(ii) For all $f: n \rightarrow m, g: m \rightarrow k$ in $L, f g$ is a least upper bound of $\left\{f \Delta_{i} g \mid i \in m\right\}$.

(iii) For all $f: n \rightarrow m$ in $L, \operatorname{cl}(f)=\{g \mid g \sqsubseteq f\}$.

2) If the equivalent conditions of 1) are satisfied, then $\sigma_{1}$ is monic iff $\sqsubseteq$ is a partial order.

Summarizing the above, a bounded large Lawvere theory $L$ admits unbounded nondeterminism iff the approximation preorder on $L$ is a partial order and for all $f: n \rightarrow m$, $g: m \rightarrow k$ in $L$,

$$
f g=\bigsqcup_{i \in m} f \Delta_{i} g .
$$

Suprema of this form are preserved in the tensor.

Remark 33. There is a variant of Theorem 32 for tensoring finitary Lawvere theories with finite non-determinism, in which $n, m, k$ are finite in (ii), (iii).

Remark 34 (Equational conservativity). One may wonder whether restricting to equational logic leads to weaker conditions for conservativity, which in the equational setting will be understood as faithfulness of the tensor map $\sigma_{1}$ : $L \rightarrow L \otimes L_{\mathcal{P}}$. However, the conditions for order-theoretic conservativity of Theorem 32 turn out to be necessary already for faithfulness of $\sigma_{1}$ under the mild additional assumption that $L$ is simply ordered, i.e. given any upper bound $h$ of $A \subseteq L(n, m)$, there exist $f, g$ such that $f g$ is an upper bound of $A, f g \sqsubseteq h$, and for every $i$ there is $a \in A$ for which $f \Delta_{i} g \sqsubseteq a$. All example theories mentioned so far are simply ordered. For simply ordered theories, closed sets are closed under all existing suprema, similarly to Scott closed sets, and thus all existing suprema are preserved in the powermonad.

Example 35. All absolutely free theories $L$ with at most one constant, such as input and output, map faithfully into $L \otimes L_{\perp}$, which can be shown to admit unbounded nondeterminism by Theorem 32 (see however Remark 21).

The partial state monad $S \rightarrow\left(S \times \_\right) \perp$ admits unbounded nondeterminism, and Lemma 30 allows identifying the tensor as the nondeterministic state monad $S \rightarrow \mathcal{P}(S \times \ldots)$ (this can also be obtained from the known description of tensors with the state monad [13]).

Every finitely additive finitary Lawvere theory admits unbounded nondeterminism. Hence, adding finite nondeterminism to a finitary theory is conservative iff adding unbounded nondeterminism is conservative.

Multisets do not admit nondeterminism: the upper bound of $\{a, \perp\}$ and $\{\perp, a\}$ is not $\{a, a\}$ but $\{a\}$. Similarly, lists do not admit unbounded nondeterminism, as $[a, b]$ is not a supremum of $[a, \perp]=[a]$ and $[\perp, b]=[b]$. In both cases, already faithfulness of the tensor map fails.

\section{CONCLUSION}

We have proved the existence of tensors of large Lawvere theories for the case that one of the components is uniform. This implies in particular that one can always tensor with unbounded nondeterminism and with continuations, in the latter case improving a previous existence result [12]. We have then given a characterization of bounded theories that can be conservatively tensored with nondeterminism, which means precisely that one can assume such theories to be completely additive. Completely additive theories support a 
calculus for Kleene iteration, in generalization of classical Kleene algebra, and, e.g., admit a generalized form of the classical Fischer-Ladner encoding [19].

Neither the present work nor [18] cover tensors with finite powerset, whose existence remains an open question. Although our results already have a quite order-theoretic flavour, an important issue for future research is whether similar results can be obtained in a domain-theoretic setting, using cpo-enriched Lawvere theories. Another direction for extending our results is to generalize them to enrichment over a topos, with a view to covering presheaf-based effects such as local state [29] or name creation [15].

Acknowledgments: We gratefully acknowledge useful insights of various contributors shared through the categories mailing list, in particular Gordon Plotkin, Peter Johnstone, and Paul Levy. Erwin R. Catesbeiana has commented on inconsistent Lawvere theories.

\section{REFERENCES}

[1] E. Moggi, "Notions of computation and monads," Inf. Comput., vol. 93, pp. 55-92, 1991.

[2] B. Jacobs and E. Poll, "Coalgebras and Monads in the Semantics of Java," Theoret. Comput. Sci., vol. 291, pp. 329349, 2003.

[3] N. Papaspyrou, "Denotational semantics of ANSI C," Computer Standards and Interfaces, vol. 23, pp. 169-185, 2001.

[4] M. Shinwell and A. Pitts, "On a monadic semantics for freshness," Theoret. Comput. Sci., vol. 342, pp. 28-55, 2005.

[5] W. Harrison, "The essence of multitasking," in Algebraic Methodology and Software Technology, AMAST 2006, ser. LNCS, vol. 4019. Springer, 2006, pp. 158-172.

[6] P. Wadler, "How to declare an imperative," ACM Comput. Surveys, vol. 29, pp. 240-263, 1997.

[7] E. Moggi and A. Sabry, "Monadic encapsulation of effects: A revised approach (extended version)," J. Funct. Prog., vol. 11, pp. 591-627, 2001.

[8] W. Lawvere, "Functorial semantics of algebraic theories," Proc. Natl. Acad. Sci. USA, vol. 50, no. 5, pp. 869-872, 1963.

[9] G. Plotkin and J. Power, "Notions of computation determine monads," in Foundations of Software Science and Computation Structures, FoSSaCS 2002, ser. LNCS, vol. 2303. Springer, 2002, pp. 342-356.

[10] P. Cenciarelli and E. Moggi, "A syntactic approach to modularity in denotational semantics," in Category Theory and Computer Science, CTCS 1993, 1993.

[11] S. Liang, P. Hudak, and M. Jones, "Monad transformers and modular interpreters," in Principles of Programming Languages, POPL 95. ACM Press, 1995, pp. 333-343.

[12] M. Hyland, P. Levy, G. Plotkin, and J. Power, "Combining algebraic effects with continuations," Theoret. Comput. Sci., vol. 375, pp. $20-40,2007$.
[13] M. Hyland, G. Plotkin, and J. Power, "Combining effects: Sum and tensor," Theoret. Comput. Sci., vol. 357, pp. 70-99, 2006.

[14] J. Power and O. Shkaravska, "From comodels to coalgebras: State and arrays," in Coalgebraic Methods in Computer Science, CMCS 2004, ser. ENTCS, vol. 106. Elsevier, 2004, pp. 297-314.

[15] I. Stark, "Free-algebra models for the $\pi$-calculus," Theoret. Comput. Sci., vol. 390, pp. 248-270, 2008.

[16] R. Tix, K. Keimel, and G. Plotkin, "Semantic domains for combining probability and non-determinism," ENTCS, vol. 222, pp. 3-99, 2009.

[17] D. Varacca and G. Winskel, "Distributing probability over non-determinism," Math. Struct. Comput. Sci., vol. 16, pp. 87-113, 2006.

[18] S. Goncharov and L. Schröder, "A counterexample to tensorability of effects,” DFKI, Tech. Rep., 2011.

[19] M. Fischer and R. Ladner, "Propositional dynamic logic of regular programs," J. Comput. Sys. Sci., 1979.

[20] G. Plotkin, "LCF considered as a programming language," Theoret. Comput. Sci., vol. 5, pp. 223-255, 1977.

[21] E. Dubuc, Kan Extensions in Enriched Category Theory, ser. LNM. Springer, 1970, vol. 145.

[22] S. Abramsky and A. Jung, "Domain theory," in Handbook of Logic in Computer Science. Oxford University Press, 1994, vol. 3, pp. 1-168.

[23] M. Droste, W. Kuich, and H. Vogler, Eds., Handbook of Weighted Automata. Springer, 2009.

[24] S. Peyton-Jones, Ed., Haskell 98 Language and Libraries The Revised Report. Cambridge University Press, 2003, also: J. Funct. Prog. 13 (2003).

[25] M. Hyland and J. Power, "The category theoretic understanding of universal algebra: Lawvere theories and monads," ENTCS, vol. 172, pp. 437-458, 2007.

[26] S. Goncharov, "Kleene monads," Ph.D. dissertation, Universität Bremen, 2010.

[27] S. Goncharov, L. Schröder, and T. Mossakowski, "Kleene monads: handling iteration in a framework of generic effects," in Algebra and Coalgebra in Computer Science, CALCO 2009, ser. LNCS, vol. 5728. Springer, 2009, pp. 18-33.

[28] B. Eckmann and P. Hilton, "Group-like structures in general categories I - multiplications and comultiplications," Math. Ann., vol. 145, pp. 227-255, 1962.

[29] J. Power, "Semantics for local computational effects," in Mathematical Foundations of Programming Semantics, MFPS 2006, ser. ENTCS, vol. 158, 2006, pp. 355-371. 


\section{APPENDIX}

(In the appendix, we use the term Lawvere theory to mean large Lawvere theory.)

\section{A. Proof of Theorem 10.}

The proof is by explicit syntactic construction of the tensor product $L=L_{1} \otimes L_{2}$. To begin, we define a (not necessarily locally small) category $\mathbf{C}$ on top of $L_{1}, L_{2}$ as follows. For $f \in \operatorname{Hom}_{L_{2}}(k, m)$ and $g \in \operatorname{Hom}_{L_{1}}(n, k)$ let $f *_{k} g$ be a synonym for the pair $\langle f, g\rangle$. We agree to omit the subscript at $*$ if it is clear from the context. We also agree that $*$ binds weaker than composition. Let us define objects of $\mathbf{C}$ to be sets and morphisms from $\operatorname{Hom}_{\mathbf{C}}(n, m)$ to be finite paths

$$
\left(f_{1} * g_{1}|\ldots| f_{k} * g_{k}\right)
$$

adhering to the typing constraints: $n$ is the source of $g_{k}, m$ is the target of $f_{1}$, and for $i=1, \ldots, k-1$, the source of $g_{i+1}$ is the target of $f_{i}$. We often omit brackets for one-element paths.

The identity morphisms of $\mathbf{C}$ are the empty paths, and composition is concatenation of paths. Clearly, $\mathbf{C}$ is a category. On every hom-set of $\mathbf{C}$ we define an equivalence relation $\sim$ as the equivalence generated by the clauses

$$
\begin{aligned}
(\ldots|[\mathrm{id}] *[\mathrm{id}]| & \ldots) \\
(\ldots|f[e] * g| \ldots \mid & \sim(\ldots)
\end{aligned}
$$

and

$$
\begin{aligned}
\left(\ldots\left|f *\left(n^{\prime} \otimes g\right)\right|\right. & \left.\left(f^{\prime} \otimes m\right) * g^{\prime} \mid \ldots\right) \\
& \left.\sim 0 \ldots\left|f\left(f^{\prime} \otimes m^{\prime}\right) *(n \otimes g) g^{\prime}\right| \ldots\right)
\end{aligned}
$$

where $f^{\prime}: n \rightarrow n^{\prime}$ and $g: m \rightarrow m^{\prime}$. By construction, is a congruence on $\mathbf{C}$, so that we have a quotient category $\mathrm{C} / \sim$. Using the fact that $L_{2}$ is uniform, we show that every morphism of $\mathbf{C} / \sim$ has a representative of the form

$$
\left(f * g \mid \mathrm{c}_{L_{2}}^{n} *[\mathrm{id}]\right) \text {. }
$$

To that end, let us take any morphism $f$ of $\mathbf{C}$. By attaching sufficiently many elements [id]* [id] in the end of $f$ we ensure that its length is at least 2 and its last element is [id]* [id]. Then we successively apply the following reduction sequence, whose net effect is length-decreasing, as long as possible:

$$
\begin{aligned}
& \left(f_{1} * g_{1}\left|f_{2} * g_{2}\right| \ldots\right) \\
& \sim\left(f_{1} * g_{1}\left|\left(\hat{f}_{2} \otimes m_{2}\right)\left[u_{f_{2}}\right] \mathrm{c}_{L_{2}}^{k_{1}} * g_{2}\right| \ldots\right) \\
& =\left(f_{1} * g_{1} \mid\left(\hat{f}_{2} \otimes m_{2}\right)\left(\left[u_{f_{2}}\right] \mathrm{c}_{L_{2}}^{k_{1}} \otimes 1\right)\right. \\
& \text { * } \left.\left(k_{1} \otimes[\mathrm{id}]\right) g_{2} \mid \ldots\right) \\
& \sim\left|f_{1} * g_{1}\right|\left(\hat{f}_{2} \otimes m_{2}\right) *\left(\left(s \times m_{2}\right) \otimes[\mathrm{id}]\right) \mid \\
& \left.\left(\left[u_{f_{2}}\right] c_{L_{2}}^{k_{1}} \otimes 1\right) * g_{2} \mid \ldots\right) \\
& =\left(f_{1} * g_{1}\left|\left(\hat{f}_{2} \otimes m_{2}\right) *[\mathrm{id}]\right|\left[u_{f_{2}}\right] c_{L_{2}}^{k_{1}} * g_{2} \mid \ldots\right)
\end{aligned}
$$

$$
\begin{aligned}
& \left.\sim\left|f_{1} * g_{1}\right|\left(\hat{f}_{2} \otimes m_{2}\right) *\left[u_{f_{2}}\right]\left|c_{L_{2}}^{k_{1}} * g_{2}\right| \ldots\right\rangle \\
& \left.=\emptyset f_{1} *\left(1 \otimes g_{1}\right)\left|\left(\hat{f}_{2} \otimes m_{2}\right) *\left[u_{f_{2}}\right]\right| c_{L_{2}}^{k_{1}} * g_{2}|\ldots\rangle\right) \\
& \left.\sim 0 f_{1}\left(\hat{f}_{2} \otimes k_{1}\right) *\left(s \otimes g_{1}\right)\left[u_{f_{2}}\right]\left|c_{L_{2}}^{k_{1}} * g_{2}\right| \ldots\right) .
\end{aligned}
$$

Here, $f_{i}: k_{i} \rightarrow m_{i}, g_{i}: n_{i} \rightarrow k_{i}$ and $\hat{f}_{2}: s \rightarrow 1$. At the last step of the reduction we obtain a pair of the form (2).

Let us define $I:$ Set $^{o p} \rightarrow \mathbf{C} / \sim$ by putting $I(n)=n$ for every set $n$ and $I(e)=[e] *$ id] for every set-function $e: n \rightarrow m$. We would like to prove that $I$ weakly preserves small products, i.e. the families $I\left(\kappa_{i}\right): \sum_{i} n_{i} \rightarrow n_{i}$ define weak small products in $\mathbf{C} / \sim$. Let $f_{i}: m \rightarrow n_{i}$ be a family of morphisms in $\mathrm{C} / \sim$. First we consider a special case when every $f_{i}$ is presentable by a one-element path, e.g. $f_{i} \sim$ $g_{i} *_{k_{i}} h_{i}$. Since $L_{2}$ has all small products, there exists a morphism $h: m \rightarrow \sum_{i} k_{i}$ of $L_{2}$ such that for every $i$, $h_{i}=\left[\kappa_{i}\right] h$. Analogously, since $L_{1}$ has all small products, there exists a morphism $g: \sum_{i} k_{i} \rightarrow \sum_{i} n_{i}$ of $L_{1}$ such that for every $i, g_{i}\left[\kappa_{i}\right]=\left[\kappa_{i}\right] g$. The equality $f_{i}=I\left(\kappa_{i}\right)(g * h)$, characterizing weak products, now follows from the diagram

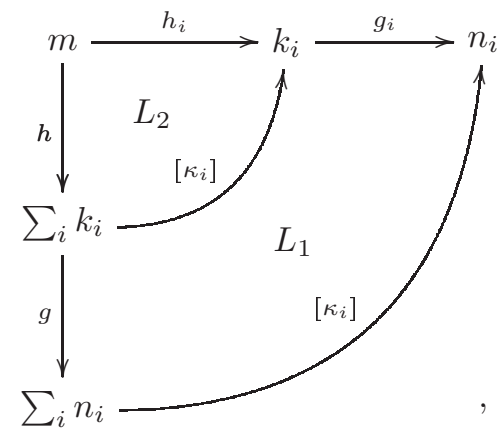

whose two cells are commuting in $L_{1}$ and in $L_{2}$ respectively. In general, the $f_{i}$ might not be presentable by one-element paths, but as we have argued above, they must be presentable by paths of length 2 . In particular, for every $i, f_{i} \sim g_{i} h_{i}$ where both $g_{i}: k_{i} \rightarrow n_{i}$ and $h_{i}: m \rightarrow k_{i}$ are one-element. As we have proved, there exists $h$ such that for every $i$, $h_{i}=I\left(\kappa_{i}\right) h$. On the other hand, every $g_{i} I\left(\kappa_{i}\right)$ is easily seen to be equivalent to a one-element path and therefore, there exists $g$ such that for every $i, g_{i} I\left(\kappa_{i}\right)=I\left(\kappa_{i}\right) g$. We have thus: $f_{i} \sim g_{i} h_{i} \sim g_{i} I\left(\kappa_{i}\right) h \sim I\left(\kappa_{i}\right) g h$ and we are done.

Let us prove that $\mathbf{C} / \sim$ is locally small. Since every morphism of $\mathbf{C}$ has the form (2), every hom-set of $\mathbf{C} / \sim$ has at most as many equivalence classes as there are nonequivalent morphisms (2) in the corresponding hom-set of C. Let us fix some pair $\left(f *_{k} g \mid c_{L_{2}}^{n} *[\mathrm{id}]\right) \in \operatorname{Hom}_{\mathbf{C}}(n, m)$. Let $c=\operatorname{Hom}_{L_{2}}(0,1)$. By local smallness of $L_{2}, s=$ $\operatorname{Hom}_{L_{2}}(n+c, 1)$ is a set, and thus $s \in \mathrm{Ob}\left(L_{1}\right)$. For every $i \in k, g_{i}=\left[\kappa_{i}\right] g$ belongs to $\operatorname{Hom}_{L_{2}}(n+c, 1)$ and we denote by $u: k \rightarrow s$ the induced index transformation. Let $h: n+c \rightarrow s$ be the tupling morphism for the whole family $\operatorname{Hom}_{L_{2}}(n+c, 1)$. Then

$$
\left(f *_{k} g \mid \mathrm{c}_{L_{2}}^{n} *[\mathrm{id}]\right) \sim\left(f *_{k}[u] h \mid \mathrm{c}_{L_{2}}^{n} *[\mathrm{id}]\right)
$$




$$
\sim\left(f[u] *_{s} h \mid \mathrm{c}_{L_{2}}^{n} *[\mathrm{id}]\right) .
$$

We have thus shown that every morphism of $\operatorname{Hom}_{\mathbf{C} / \sim}(n, m)$ has a representative in the set $\operatorname{Hom}_{L_{1}}(m, s) \times \operatorname{Hom}_{L_{2}}(n, s)$ and hence $\operatorname{Hom}_{\mathbf{C} / \sim}(n, m)$ is also a set.

Let $\approx$ be the smallest congruence on $\mathbf{C}$, containing $\sim$ and closed under the rule:

$$
\forall i .\left[\kappa_{i}\right] f \approx\left[\kappa_{i}\right] g \Longrightarrow f \approx g .
$$

We then have a canonical functor $\mathrm{C} / \sim \rightarrow \mathrm{C} / \approx$, which equips $\mathbf{C} / \approx$ with all small weak products, which due to $(3)$ are in fact products. By postcomposing $I: \mathbf{S e t}^{o p} \rightarrow \mathbf{C} / \sim$ with the canonical projection, we obtain a product preserving functor $I: \operatorname{Set}^{o p} \rightarrow \mathbf{C} / \approx$, so that $L=\mathbf{C} / \approx$ is a Lawvere theory. We will be done once we show that $L=L_{1} \otimes L_{2}$. We define functors $\sigma_{i}: L_{i} \rightarrow L$ by

$$
\sigma_{1}(f)=f *[\mathrm{id}], \quad \sigma_{2}(g)=[\mathrm{id}] * g
$$

(omitting equivalence class formation from the notation). The following calculation ensures commutativity of $\sigma_{1}$ and $\sigma_{2}$ :

$$
\begin{aligned}
\left(\sigma_{2}(g)\right. & \left.\otimes n^{\prime}\right)\left(m \otimes \sigma_{1}(f)\right) \\
& =\left(0[\mathrm{id}] * g D \otimes n^{\prime}\right)(m \otimes(f *[\mathrm{id}])) \\
& =\left([\mathrm{id}] * g \otimes n^{\prime} D(m \otimes f *[\mathrm{id}])\right. \\
& =[\mathrm{id}]\left(m^{\prime} \otimes f\right) *(g \otimes n)[\mathrm{id}] \\
& =\left(m^{\prime} \otimes f\right) *(g \otimes n) \\
& =\left(m^{\prime} \otimes f *[\mathrm{id}] D Q[\mathrm{id}] * g \otimes n D\right. \\
& =\left(m^{\prime} \otimes(f *[\mathrm{id}] D)(0[\mathrm{id}] * g D \otimes n)\right. \\
& =\left(m^{\prime} \otimes \sigma_{1}(f)\right)\left(\sigma_{2}(g) \otimes n\right) .
\end{aligned}
$$

Finally, let $L^{\prime}$ be another Lawvere theory equipped with a pair of commuting morphisms $\alpha_{i}: L_{i} \rightarrow L^{\prime}$. We define a morphism of categories $\alpha: \mathbf{C} \rightarrow L^{\prime}$ to be identity on objects and by the equations $\alpha 0$ D $=$ id and

$$
\begin{aligned}
& \left.\alpha\left|f_{1} * g_{1}\right| \ldots \mid f_{k} * g_{k}\right)= \\
& \alpha_{1}\left(f_{1}\right) \alpha_{2}\left(g_{1}\right) \ldots \alpha_{1}\left(f_{k}\right) \alpha_{2}\left(g_{k}\right)
\end{aligned}
$$

on morphisms. It is straightforward to verify by definition that $f \approx g$ implies $\alpha(f)=\alpha(g)$. Therefore, by the characteristic property of the quotient category, $\alpha$ lifts to a morphism of Lawvere theories $\alpha: L \rightarrow L^{\prime}$. It is again easy to verify that for $i=1,2, \alpha_{i}=\alpha \sigma_{i}$. Uniqueness of $\alpha$ is clear. Therefore $L$ is indeed a tensor product of $L_{1}$ and $L_{2}$ and we are done.

\section{B. Proof of Proposition 20}

1) Let for every $\Gamma \triangleright t: T A \times T A, h(t)=\mathrm{fst}(t)+\operatorname{snd}(t)$. Then

if $b$ then $p$ else $q$

$$
\begin{aligned}
& =\text { case } b \text { of inl } \star \mapsto p ; \operatorname{inr} \star \mapsto q \\
& =\text { case } b \text { of inl } \star \mapsto h\langle p, \perp\rangle ; \operatorname{inr} \star \mapsto h\langle\perp, q\rangle
\end{aligned}
$$

$$
\begin{aligned}
= & h(\text { case } b \text { of inl } \star \mapsto\langle p, \perp\rangle ; \operatorname{inr} \star \mapsto\langle\perp, q\rangle) \\
= & h(\text { case } b \text { of inl } \star \mapsto p ; \operatorname{inr} \star \mapsto \perp, \\
& \quad \text { case } b \text { of inl } \star \mapsto \perp ; \operatorname{inr} \star \mapsto q) \\
= & \text { do }(\text { case } b \text { of inl } \star \mapsto \text { ret } \star ; \operatorname{inr} \star \mapsto \perp) ; p+ \\
& \text { do }(\text { case } b \text { of inl } \star \mapsto \perp ; \operatorname{inr} \star \mapsto \text { ret } \star) ; q \\
= & \text { do }(\text { case } b \text { of inl } \star \mapsto \text { ret } \star ; \operatorname{inr} \star \mapsto \perp) ; p+ \\
& \text { do }(\text { case } \neg b \text { of inl } \star \mapsto \text { ret } \star ; \operatorname{inr} \star \mapsto \perp) ; q \\
= & \text { do } b ? ; p+\text { do }(\neg b) ? ; q
\end{aligned}
$$

and we are done.

2) First, note that by Lemma 24 , $\sqsubseteq$ coincides with $\leqslant$. By part (i), we need to show that

$$
\text { do } x \leftarrow\left(\text { init } x \leftarrow \operatorname{ret} x \text { in }(\text { do } b ? ; p)^{\star}\right) ;(\neg b) \text { ?; ret } x
$$

is the least fixed point of

$$
q \mapsto \text { do } b ? ; x \leftarrow p ; q+\operatorname{do}(\neg b) \text { ?; ret } x .
$$

First observe that (4) is a fixed point of (5):

$$
\begin{aligned}
\text { do } b ? ; & \leftarrow x ;\left(\text { do } x \leftarrow\left(\text { init } x \leftarrow \operatorname{ret} x \operatorname{in}(\text { do } b ? ; p)^{\star}\right) ;\right. \\
& (\neg b) ? ; \operatorname{ret} x)+\operatorname{do}(\neg b) ? ; \operatorname{ret} x \\
= & \text { do } x \leftarrow(\operatorname{do} b ? ; x \leftarrow p ; \\
& \text { init } \left.x \leftarrow \operatorname{ret} x \operatorname{in}(\operatorname{do} b ? ; p)^{\star}+\operatorname{ret} x\right) ;(\neg b) ? ; \text { ret } x \\
= & \text { do } x \leftarrow\left(\text { init } x \leftarrow(\operatorname{do} b ? ; p) \operatorname{in}(\operatorname{do} b ? ; p)^{\star}\right. \\
& +\operatorname{ret} x) ;(\neg b) ? ; \operatorname{ret} x \\
= & \text { do } x \leftarrow\left(\text { init } x \leftarrow \operatorname{ret} x \operatorname{in}(\operatorname{do} b ? ; p)^{\star}\right) ;(\neg b) ? ; \operatorname{ret} x .
\end{aligned}
$$

In order to show that (4) is the least fixed point, suppose $q$ is some other fixed point of (5). Then

$$
\begin{aligned}
& \text { do } b ? ; x \leftarrow p ; q \leqslant q \\
& \text { do }(\neg b) ? ; \text { ret } x \leqslant q
\end{aligned}
$$

From the former inequality, by (ind $\mathbf{i n}_{2}$ ):

$$
\text { do } x \leftarrow\left(\text { init } x \leftarrow \operatorname{ret} x \operatorname{in}(\text { do } b ? ; p)^{\star}\right) ; q \leqslant q
$$

from which we conclude by the latter inequality,

$$
\text { do } \left.x \leftarrow \text { (init } x \leftarrow \operatorname{ret} x \text { in }(\text { do } b \text { ?; } p)^{\star}\right) ;(\neg b) \text { ?; ret } x \leqslant q \text {. }
$$

Therefore (4) is indeed the least fixed point of (5) and the proof is thus completed.

\section{Proof of Lemma 24}

To prove that $\sqsubseteq$ is contained in $\preceq$, it suffices to show that $\preceq$ has the closure properties defining $\sqsubseteq$. By definition, $\preceq$ has $\perp$ as bottom. To see that $\preceq$ is closed under $\left(\pi_{\sqsubseteq}\right)$, let $f, g: n \rightarrow m$ in $L$ such that $\left[\kappa_{i}\right] f \preceq\left[\kappa_{j}\right] g$ for all $i \in m$. By definition, this means that $\left[\kappa_{i}\right] f+\left[\kappa_{j}\right] g\left[\kappa_{i}\right](f+g)=\left[\kappa_{j}\right] g$ for all $i$, so that $f+g=g$, i.e. $f \preceq g$; since by definition, composition is monotone w.r.t. $\preceq$, it follows that $h f \preceq h g$ for $h: m \rightarrow k$.

To show that, conversely, $\preceq$ is contained in $\sqsubseteq$, let $f \preceq g$. Then $f=f+\perp \sqsubseteq f+g=g$. 


\section{A Direct Construction of the Nonempty Powermonad}

To pave the ground for the direct construction of the powermonad, i.e. the proof of Theorem 27 , we describe the direct construction of the nonempty powermonad, i.e. tensoring with non-blocking unbounded nondeterminism.

We need a preliminary lemma to ease the proof of the tensor equation.

Lemma 36. In the notation of Definition 4, the tensor equation reduces to the case $m_{1}=m_{2}=1$.

Proof: We prove the general case as follows: To check commutation of the requisite diagram for arbitrary $m_{1}, m_{2}$, it suffices to check commutation for all postcompositions with the product projections $\pi_{i j}=[(i, j)]: m_{1} \times m_{2} \rightarrow 1$ for $i \in m_{1}, j \in m_{2}$ (where $(i, j): 1 \rightarrow m_{1} \times m_{2}$ denotes the obvious constant map). Note that $\pi_{i j}=\pi_{i}\left(m_{1} \otimes \pi_{j}\right)=$ $\pi_{j}\left(\pi_{i} \otimes m_{2}\right)$ where $\pi_{i}: m_{1} \rightarrow 1$ and $\pi_{j}: n_{1} \rightarrow 1$ are product projections. Therefore $\pi_{i j}\left(f_{1} \otimes m_{2}\right)=\pi_{i}\left(m_{1} \otimes\right.$ $\left.\pi_{j}\right)\left(f_{1} \otimes m_{2}\right)=\pi_{i}\left(f_{1} \otimes \pi_{j}\right)=\left(\pi_{i} f_{1} \otimes \pi_{j}\right)$ and $\pi_{i j}\left(m_{1} \otimes\right.$ $\left.f_{2}\right)=\pi_{j}\left(\pi_{i} \otimes m_{2}\right)\left(m_{1} \otimes f_{2}\right)=\pi_{j}\left(\pi_{i} \otimes f_{2}\right)=\pi_{i} \otimes\left(\pi_{j} f_{2}\right)$. (Note here that for $f: n \rightarrow m$ and a map $e: k \rightarrow l$, $f \otimes[e]: n \times l \rightarrow m \times k$ is definable as the morphism into the $k$-fold product $m \times k$ whose postcomposition with the $j$-th projection $m \times k \rightarrow m(j \in k)$ is $f \pi_{e(j)}$, where $\pi_{e(j)}$ is the $e(j)$-th product projection $n \times l \rightarrow n$.) Next note that $\left(\pi_{i} f_{1} \otimes \pi_{j}\right)\left(n_{1} \otimes f_{2}\right)=\pi_{i} f_{1}\left(n_{1} \otimes \pi_{j} f_{2}\right)$ and $\left(\pi_{i} \otimes \pi_{j} f_{2}\right)\left(f_{1} \otimes\right.$ $\left.n_{2}\right)=\pi_{j} f_{2}\left(\pi_{i} f_{1} \otimes n_{2}\right)$, so that we are done by commutation of

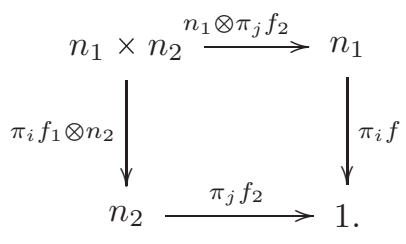

Let $L$ be a Lawvere theory. We give a construction of the tensor $T=L \otimes L_{\mathcal{P}^{*}}$. We begin by constructing a category $T_{0}$ with an identity-on-objects functor $I_{0}:$ Set $\rightarrow T_{0}$, with the same notation as for Lawvere theories, with the following properties:

- $I_{0}$ maps products to weak products;

- $T_{0}$ has functors $F^{0}: L \rightarrow T_{0}, F_{\mathcal{P}^{*}}^{0}: L_{\mathcal{P}^{*}} \rightarrow T_{0}$ that commute with the respective functors from Set

That is, $T_{0}$ will fail to be the tensor $L \otimes L_{\mathcal{P}^{*}}$ on two counts: tupling morphisms need not be unique in $T_{0}$, and the two functors from the component theories into $T_{0}$ need not commute. One of the surprises in the construction is that repairing the first defect will remedy also the second one.

Morphisms $n \rightarrow m$ in $T_{0}$ are just nonempty sets of $L$ morphisms. Composition is defined by $A B=\{a b \mid a \in$ $A, b \in B\}$; identities are singleton sets $\left\{\operatorname{id}_{n}\right\}$. The functor $F^{0}: L \rightarrow T_{0}$ maps a morphism $f$ to the singleton $\{f\}$. We then define $I_{0}$ as the composite Set $\rightarrow L \rightarrow T_{0}$. The tupling $\left\langle A_{i}\right\rangle: n \rightarrow \sum m_{i}$ of $k T_{0}$-morphisms $A_{i}: n \rightarrow m_{i}$ is defined as

$$
\left\langle A_{i}\right\rangle=\left\{\left\langle f_{i}\right\rangle \mid f_{i} \in A_{i} \text { for all } i\right\},
$$

where $\left\langle f_{i}\right\rangle$ denotes tupling in $L$. We regard morphisms $n \rightarrow$ $m$ in $L_{\mathcal{P}^{*}}$ as $m$-tuples $\left(A_{i}\right)$ of nonempty subsets of $n$. Then the functor $F_{\mathcal{P}^{*}}^{0}: L_{\mathcal{P}^{*}} \rightarrow T_{0}$ maps $\left(A_{i}\right)$ to

$$
F_{\mathcal{P}^{*}}^{0}\left(\left(A_{i}\right)\right)=\left\{[e] \mid e: m \rightarrow n, e(i) \in A_{i} \text { for all } i\right\} .
$$

In the special case $m=1$, in which case a morphism $n \rightarrow m$ is just a single subset $A \subseteq n$, note that $F_{\mathcal{P}^{*}}^{0}(A)=\left\{\left[\kappa_{i}\right] \mid\right.$ $i \in A\}$.

This completes the definition of $T_{0}$. We need to check a few properties:

- The composite Set $\rightarrow L_{\mathcal{P}^{*}} \rightarrow T_{0}$ coincides with $I_{0}$. To see this, let $e: m \rightarrow n$ be a map. In $L_{\mathcal{P}^{*}},[e]: n \rightarrow m$ is the $m$-tuple $(\{e(i)\})_{i \in m}$. Under $F_{\mathcal{P}^{*}}^{0}$, this becomes the set

$$
\{[\bar{e}] \mid \bar{e}: m \rightarrow n, \bar{e}(i) \in\{e(i)\} \text { for all } i\}=\{[e]\} \text {. }
$$

- The tupling morphisms project back to their components: For $A_{i}: n \rightarrow m_{i}$ in $T_{0}$, we have

$$
\begin{aligned}
\pi_{j}\left\langle A_{i}\right\rangle=\left\{\pi_{j}\left\langle f_{i}\right\rangle \mid\left(f_{i}\right) \in \prod\right. & \left.A_{i}\right\}= \\
& \left\{f_{j} \mid\left(f_{i}\right) \in \prod A_{i}\right\}=A_{j},
\end{aligned}
$$

as the $A_{i}$ are nonempty (note that this requires the axiom of choice).

We now proceed to repair the mentioned defects by quotienting $T_{0}$ by an appropriate equivalence relation. We define the relation $\approx$ as the smallest reflexive transitive relation closed under the infinitary rule

$$
\text { ( } \pi) \frac{\forall i \cdot\left[\kappa_{i}\right] A \approx\left[\kappa_{i}\right] B}{C A \approx C B} .
$$

Lemma 37. The relation $\approx$ satisfies the following properties.

$1) \approx$ is symmetric.

$2) \approx$ is a congruence w.r.t. composition.

3) $\approx$ is a congruence w.r.t. tupling.

\section{Proof:}

1) Put $\approx^{s}=\approx \cap \approx^{-}$, where $\cdot-$ denotes the inverse relation. Clearly, $\approx^{s}$ is reflexive and transitive. Moreover, $\approx^{s}$ is easily seen to be closed under $(\pi)$. Consequently, $\approx \subseteq \approx^{s}$, so that $\approx$ is symmetric.

2) Let

$$
A \approx^{c} B \Longleftrightarrow \forall L, R . L A R \approx L B R .
$$

Then $\approx^{c}$ is clearly reflexive and transitive. Moreover, $\approx^{c}$ is closed under $(\pi)$ : if $\left[\kappa_{i}\right] A \approx^{c}\left[\kappa_{i}\right] B$ for all $i$, then in particular $\left[\kappa_{i}\right] A R \approx\left[\kappa_{i}\right] B R$ for all $i, R$ and hence $L A R \approx$ $L B R$ for all $L, R$. Therefore, $\approx^{c}$ contains $\approx$, so that $\approx$ is a congruence. 
3) Let $A_{i}, B_{i}: n \rightarrow m_{i}$ and $A_{i} \approx B_{i}$ for $i \in k$. To prove $\left\langle A_{i}\right\rangle \approx\left\langle B_{i}\right\rangle$, we have to show $\left[\kappa_{(i, j)}\right]\left\langle A_{i}\right\rangle \approx\left[\kappa_{(i, j)}\right]\left\langle B_{i}\right\rangle$ for $i \in k, j \in m_{i}$ (i.e. $\left.(i, j) \in \sum_{i \in k} m_{i}\right)$. Now $\kappa_{(i, j)}=\left(\kappa_{j} \times\right.$ $\left.\operatorname{id}_{k}\right) \iota_{i}$, where $\iota_{i}: m_{i} \rightarrow \sum m_{i}$ is the coproduct embedding (and hence $\left[\iota_{i}\right]$ is a weak product projection). Thus, we have $\left[\kappa_{(i, j)}\right]\left\langle A_{i}\right\rangle=\left[\kappa_{j}\right] A_{i} \approx\left[\kappa_{j}\right] B_{i}=\left[\kappa_{(i, j)}\right]\left\langle B_{i}\right\rangle$, using the assumption $A_{i} \approx B_{i}$ and the fact that $\approx$ is congruent w.r.t. composition.

A last observation that needs to be made is that the tensor law holds in $T_{0}$ modulo $\approx$ : Let $A \subseteq m$, corresponding to the morphism $\bar{A}=\left\{\left[\kappa_{i}\right] \mid i \in A\right\}: m \rightarrow 1$ in $T_{0}$, and let $f: n \rightarrow 1$ in $L$ (identified with a singleton in $T_{0}$ ). We have to show that the diagram

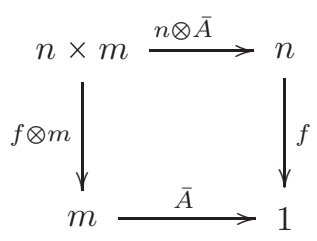

commutes. Now we have

$$
\begin{aligned}
n \otimes \bar{A} & =\left\langle\bar{A}\left[\kappa_{i} \times \mathrm{id}_{m}\right]\right\rangle_{i \in n} \\
& =\left\langle\left\{\left[\left(\kappa_{i} \times \mathrm{id}_{m}\right) \kappa_{j}\right] \mid j \in A\right\}\right\rangle_{i \in n} \\
& =\left\langle\left\{\left[\kappa_{(i, j)}\right] \mid j \in A\right\}\right\rangle_{i \in n} \\
& =\left\{\left\langle\left[\kappa_{\left(i, j_{i}\right)}\right]\right\rangle_{i \in n} \mid\left(j_{i}\right) \in A^{n}\right\}
\end{aligned}
$$

and hence

$$
f(n \otimes \bar{A})=\left\{f\left\langle\left[\kappa_{\left(i, j_{i}\right)}\right]\right\rangle_{i \in n} \mid\left(j_{i}\right) \in A^{n}\right\} .
$$

On the other hand, we have

$$
\begin{aligned}
\bar{A}(f \otimes m) & =\bar{A}\left\langle f\left[\mathrm{id}_{n} \times \kappa_{j}\right]\right\rangle_{j \in m} \\
& =\left\{\left[\kappa_{j}\right]\left\langle f\left[\mathrm{id}_{n} \times \kappa_{j}\right]\right\rangle_{j \in m} \mid j \in A\right\} \\
& \left\{f\left[\operatorname{id}_{n} \times \kappa_{j} \mid j \in A\right\}\right.
\end{aligned}
$$

so that equivalence of the two sides follows from

$$
\left\{\left[\operatorname{id}_{n} \otimes \kappa_{j}\right] \mid j \in A\right\} \approx\left\{\left\langle\kappa_{\left(i, j_{i}\right)}\right\rangle_{i \in n} \mid\left(j_{i}\right) \in A^{n}\right\} .
$$

To prove (7), we compare the projections along $\left[\kappa_{i}\right], i \in$ $n$, on both sides, and thus reduce the goal to the evident equality

$$
\begin{aligned}
\left\{\left[\kappa_{i}\right]\left[\operatorname{id}_{n} \times \kappa_{j}\right] \mid j \in A\right\} & =\left\{\left[\kappa_{(i, j)} \mid j \in A\right]\right\} \\
& =\left\{\left[\kappa_{\left(i, j_{i}\right)}\right] \mid\left(j_{i}\right) \in A^{n}\right\} .
\end{aligned}
$$

We have thus shown that $T=T_{0} / \approx$ is a candidate for the tensor product of $L$ and $\mathcal{P}^{*}$. It remains to prove the universal property. Thus, let $S$ be a further candidate, i.e. a Lawvere theory with maps $G: L \rightarrow S, G_{\mathcal{P}^{*}}: L_{\mathcal{P}^{*}} \rightarrow S$ such that the tensor law is satisfied for $G$ and $G_{\mathcal{P}^{*}}$. We define a functor $\bar{G}: T \rightarrow S$ as follows. In preparation, we note that every subset $A \subseteq n$ of some set $n$ is a morphism $n \rightarrow 1$ in $L_{\mathcal{P}^{*}}$, whose image under $G_{\mathcal{P}^{*}}$ we denote by $(A \subseteq n): n \rightarrow 1$.
In particular, for a morphism $A: n \rightarrow m$ in $T_{0}$ we have $\hat{A}:=(A \subseteq L(n, m)): L(n, m) \rightarrow 1$ in $S$. Moreover, we have for each set $n$ a morphism

$$
s_{n}=G\left(\langle f\rangle_{f \in L(n)}\right): n \rightarrow L(n)
$$

in $S$, where we denote by $L(n)$ the action of the monad induced by $L$, i.e. simply $L(n)=L(n, 1)$. We then define a functor $\bar{G}_{0}: T_{0} \rightarrow S$ by putting, for $A: n \rightarrow 1$ in $T_{0}$,

$$
\bar{G}_{0}(A)=\hat{A} s_{n} \text {. }
$$

In general, we then put

$$
\bar{G}_{0}(A)=\left\langle G\left(\left[\kappa_{i}\right] A\right)\right\rangle_{i \in m}
$$

for $A: n \rightarrow m$ in $T_{0}$ (noting that this agrees with the previous definition in case $m=1$ ).

To establish the requisite properties of $\bar{G}_{0}$, we need the following lemma.

Lemma 38. Let $A \subseteq n, B \subseteq m$, and let $e: n \rightarrow m$ such that $e[A]=B$. Then

$$
(A \subseteq n)[e]=(B \subseteq m)
$$

in $S$.

Proof: Immediate from the corresponding equality in $L_{\mathcal{P}^{*}}$.

To begin, we now show that $\bar{G}_{0}$ preserves [_], which will then also imply that $\bar{G}_{0}$ preserves identities. Thus, let $e: n \rightarrow m$ be a map; we have to show that $\bar{G}_{0}[e]=[e]$, which by applying product projections on both sides and by definition of $\bar{G}_{0}$ immediately reduces to the case $n=1$, i.e. $e=\kappa_{j}$ for some $j \in m$. Now we have

$$
\begin{aligned}
\bar{G}_{0}\left[\kappa_{j}\right] & =\widehat{\left\{\left[\kappa_{j}\right]\right\}} s_{m} \\
& =(1 \subseteq 1)\left[\kappa_{\left[\kappa_{j}\right]}\right] s_{m} \\
& =\kappa_{j},
\end{aligned}
$$

where the second step is by Lemma 38 (applied to $\left.\kappa_{\left[\kappa_{j}\right]}[1]=\left\{\left[\kappa_{j}\right]\right\}\right)$.

The crucial point in the proof is now to establish that $\bar{G}_{0}$ preserves composition. Again, this reduces immediately to the case where the codomain of the composite is 1 . Thus, let $A: n \rightarrow 1$, and let $B: k \rightarrow n$ in $T_{0}$; put $B_{j}=\left[\kappa_{j}\right] B$ and $\nu_{j}=\lambda g .\left[\kappa_{j}\right] g: L(k, n) \rightarrow L(k)$ for $j \in n$. By Lemma 38, we then have $\hat{B}_{j}=\hat{B}\left[\nu_{j}\right]$. We start to transform $\bar{G}_{0} A \bar{G}_{0} B$ :

$$
\begin{aligned}
\bar{G}_{0} A \bar{G}_{0} B & =\hat{A} s_{n}\left\langle\hat{B}_{j} s_{k}\right\rangle_{j \in n} \\
& =\hat{A} s_{n}\left\langle\hat{B}\left[\nu_{j}\right] s_{k}\right\rangle_{j \in n} \\
& =\hat{A} s_{n}(n \otimes \hat{B})\left\langle\left[\nu_{j}\right] s_{k}\right\rangle_{j \in n} \\
& =\hat{A}(L(n) \otimes \hat{B})\left(s_{n} \otimes L(k, n)\right)\left\langle\left[\nu_{j}\right] s_{k}\right\rangle_{j \in n},
\end{aligned}
$$

using the tensor law in the last step. We proceed to analyse the right-hand subterm of the last term separately: we claim that

$$
\left.s_{n} \otimes L(k, n)\right)\left\langle\left[\nu_{j}\right] s_{k}\right\rangle_{j \in n}=G\langle f g\rangle_{f \in L(n), g \in L(k, n)} .
$$


We then note moreover that the right-hand side of (8) equals $[c] s_{k}$, where $c: L(n) \times L(k, n) \rightarrow L(k)$ is composition (this is proved by precomposing both sides with the projections $\left[\kappa_{(f, g)}\right]$ : we have $\left[\kappa_{(f, g)}\right][c] s_{k}=\left[c \kappa_{(f, g)}\right] s_{k}=\left[\kappa_{f g}\right] s_{k}=$ $G(f g)$.) We then conclude the argument by

$$
\begin{aligned}
\hat{A}(L(n) \otimes \hat{B})\left(s_{n} \otimes L(k, n)\right)\langle & {\left.\left[\nu_{j}\right] s_{k}\right\rangle_{j \in n} } \\
& =\widehat{A \times B}[c] s_{k}=\widehat{A B} s_{k}
\end{aligned}
$$

again using Lemma 38 in the last step.

It remains to prove our claim (8). We note that $n \times L(k, n)$ is the $n$-fold product of $L(k, n)$ in $S$, with projections $p_{j}=$ $[\lambda g .(j, g)]$ for all $j \in n$, and at the same time the $L(k, n)$ fold product of $n$, with projections $q_{g}=[\lambda j .(j, g)]$ for all $g \in L(k, n)$, and similarly $L(n) \times L(k, n)$ is the $L(k, n)$-fold product of $L(n)$, with projections $\bar{q}_{g}=[\lambda f .(f, g)]$ for all $g \in L(k, n)$. We then prove (8) by precomposing both sides with $\left[\kappa_{(f, g)}\right]$. We have

$$
\begin{aligned}
& {\left[\kappa_{(f, g)}\right]\left(s_{n} \otimes L(k, n)\right)\left\langle\left[\nu_{j}\right] s_{k}\right\rangle_{j \in n}} \\
& =\left[\kappa_{f}\right][\lambda f .(f, g)]\left(s_{n} \otimes L(k, n)\right)\left\langle\left[\nu_{j}\right] s_{k}\right\rangle_{j \in n} \\
& =\left[\kappa_{f}\right] \bar{q}_{g}\left(s_{n} \otimes L(k, n)\right)\left\langle\left[\nu_{j}\right] s_{k}\right\rangle_{j \in n} \\
& =\left[\kappa_{f}\right] s_{n} q_{g}\left\langle\left[\nu_{j}\right] s_{k}\right\rangle_{j \in n} \\
& =G(f) q_{g}\left\langle\left[\nu_{j}\right] s_{k}\right\rangle_{j \in n} .
\end{aligned}
$$

Thus we are done once we show that $q_{g}\left\langle\left[\nu_{j}\right] s_{k}\right\rangle_{j \in n}=G(g)$. To this end, we precompose both sides with $\left[\kappa_{j}\right]$ and calculate

$$
\begin{aligned}
& {\left[\kappa_{j}\right] q_{g}\left\langle\left[\nu_{j}\right] s_{k}\right\rangle_{j \in n}} \\
& =\left[\kappa_{j}\right][\lambda j \cdot(j, g)]\left\langle\left[\nu_{j}\right] s_{k}\right\rangle_{j \in n} \\
& =\left[\kappa_{g}\right][\lambda g \cdot(j, g)]\left\langle\left[\nu_{j}\right] s_{k}\right\rangle_{j \in n} \\
& =\left[\kappa_{g}\right] p_{j}\left\langle\left[\nu_{j}\right] s_{k}\right\rangle_{j \in n} \\
& =\left[\kappa_{g}\right]\left[\nu_{j}\right] s_{k} \\
& =\left[\kappa_{\left.\left[\kappa_{j}\right] g\right]}\right] s_{k}=G\left(\left[\kappa_{j}\right] g\right)=\left[\kappa_{j}\right] G g .
\end{aligned}
$$

This concludes the proof that $\bar{G}_{0}$ preserves composition. It is then clear that $\bar{G}_{0}$ factors through $T$, as its kernel satisfies all properties featuring in the inductive definition of $\approx$ (the kernel is, of course, reflexive and transitive, and it is closed under $(\pi)$ because tupling is unique in $S$ ). Uniqueness of the arising factorizing morphism $\bar{G}: T \rightarrow S$ is clear, because every morphism $n \rightarrow m$ in $T$ has the form $F_{\mathcal{P}^{*}}(A) F(B)$, where $B: n \rightarrow k$ in $L$ and $A: k \rightarrow n$ in $L_{\mathcal{P}^{*}}$.

Summarizing the above, we have shown that

$$
T_{0} / \approx \text { is the tensor product of } L \text { and } \mathcal{P}^{*} \text {. }
$$

One consequence of this is the following property:

Lemma 39. $\approx$ is congruent w.r.t. union.

\section{E. A Direct Construction of the Power Tensor}

We proceed to give details for Theorem 27. The construction of tensor products with the full powerset theory $L_{\mathcal{P}}$ is similar to the one for the nonempty powerset, but more involved due to the fact that the full powerset theory has a constant, $\varnothing$. The general construction of tensoring a theory $L$ with uniform theories tells us that for such a case, we have to expect a three-layered normal form that has operations of $L_{\mathcal{P}}$ on top, under this a layer of operations of $L$, and at the bottom a layer consisting not only of variables but possibly also of occurrences of $\varnothing$.

To simplify matters, we have assumed that the given theory $L$ is bounded, with the unique constant denoted $\perp$. This will in particular allow us to replace occurrences of $\varnothing$ in the bottom layer with $\perp$, thus effectively reverting to a two-layered structure.

Under the assumption that $L$ is bounded, the tensor $L \otimes L_{\mathcal{P}}$ is constructed as follows. As in the case of the nonempty power tensor, we begin by constructing a preliminary category $T_{0}$. Morphisms $n \rightarrow m$ in $T_{0}$ are (possibly empty) subsets of $L(n, m)$. Composition is pointwise, as previously; also, the definition of the embedding functors $L \rightarrow T_{0}$, $L_{\mathcal{P}} \rightarrow T_{0}$ from the component theories remains unchanged, similarly for the indexing functor Set $\rightarrow T_{0}$. The crucial difference with the nonempty powerset theory is that we have to adapt the definition of tupling to work around the basic fact that Cartesian products of sets are empty if one of the sets is empty, i.e. Cartesian products do not directly provide a faithful representation of tuples of sets as sets of tuples. Here, we exploit the fact that $\perp$ is available, and by the tensor law is equivalent to the empty set. We thus put, for $A: n \rightarrow m$ in $T_{0}$,

$$
\tilde{A}= \begin{cases}\{\perp\} & \text { if } A=\varnothing \\ A & \text { otherwise }\end{cases}
$$

and then define the tupling of a family of morphisms $A_{i}$ : $n \rightarrow m, i \in k$, by

$$
\left\langle A_{i}\right\rangle_{i \in k}=\left\{\left\langle f_{i}\right\rangle_{i \in k} \mid \forall i \in k . f_{i} \in \tilde{A}_{i}\right\} .
$$

By the calculation carried out for the nonempty case, we then have $\pi_{j}\left\langle A_{i}\right\rangle=\tilde{A}_{j}$ in $T_{0}$, and subsequent quotienting will ensure that $\tilde{A}_{j}$ becomes equal to $A_{j}$. Commutation of the tensor injections with the indexing functors is as in the nonempty case.

Next, we quotient $T_{0}$ by rectangular equivalence, i.e. the relation $\approx$ defined inductively as the smallest reflexive and transitive relation closed under rule $(\pi)$ and additionally satisfying the axiom

$$
(\perp) \quad\left\{\perp_{n, m}\right\} \approx \varnothing
$$

as well as the symmetric $\varnothing \approx\left\{\perp_{n, m}\right\}$ for all $n, m$. We have

Lemma 40. The relation $\approx$ satisfies the following properties. 
1) $\approx$ is symmetric.

2) $A \approx \tilde{A}$ for all $A$.

$3) \approx$ is a congruence w.r.t. composition.

$4) \approx$ is a congruence w.r.t. tupling.

Proof:

1) As in the nonempty case.

2) Trivial.

3) Let

$$
A \approx^{c} B \Longleftrightarrow \forall L, R . L A R \approx L B R .
$$

As in the nonempty case, $\approx^{c}$ is easily seen to be reflexive, transitive, and closed under $(\pi)$. It remains to see that $\approx^{c}$ contains all instances of $(\perp)$, i.e. (considering only one of the two symmetric cases of $(\perp))$ that for all $L, R, L\{\perp\} R \approx$ $L \varnothing R=\varnothing$. But this follows from boundedness of $L$ : for $l \in$ $L, r \in R$, we have $l \perp r=\perp$, and therefore $L\{\perp\} R=\{\perp\} \approx$ $\varnothing$. Therefore, $\approx^{c}$ contains $\approx$, so that $\approx$ is a congruence.

4) Let $A_{i}, B_{i}: n \rightarrow m_{i}$ and $A_{i} \approx B_{i}$ for $i \in k$. To prove $\left\langle A_{i}\right\rangle \approx\left\langle B_{i}\right\rangle$, we have to show $\left[\kappa_{(i, j)}\right]\left\langle A_{i}\right\rangle \approx\left[\kappa_{(i, j)}\right]\left\langle B_{i}\right\rangle$ for $i \in k, j \in m_{i}$. Now $\kappa_{(i, j)}=\left(\kappa_{j} \times \operatorname{id}_{k}\right) \iota_{i}$, where $\iota_{i}: m_{i} \rightarrow \sum m_{i}$ is the coproduct embedding. Thus, we have $\left[\kappa_{(i, j)}\right]\left\langle A_{i}\right\rangle=\left[\kappa_{j}\right] \tilde{A}_{i} \approx\left[\kappa_{j}\right] A_{i}$

poweq $\left[\kappa_{j}\right] B_{i} \approx\left[\kappa_{j}\right] \tilde{B}_{i}=\left[\kappa_{(i, j)}\right]\left\langle B_{i}\right\rangle$, using the assumption $A_{i} \approx B_{i}$, congruence w.r.t. composition, and claim 2 of this lemma.

So far, we have established that $T:=T_{0} / \approx$ is a large Lawvere theory that has theory morphisms $L \rightarrow T$ and $L_{\mathcal{P}} \rightarrow T$. To prove that $T$ is a candidate for the tensor $L \otimes L_{\mathcal{P}}$, we need to show that the tensor law holds. The argument is mostly as in the nonempty case: we need only check those cases where the empty set can occur within a tupling operation; the only case in point is where $A=\varnothing$, in the notation of (6). This case, however, is taken care of by the fact that $\varnothing \approx\{\perp\}$ and by boundedness of $L$, which ensures that for $\{\perp\}$ in place of $\bar{A}$, both paths in (6) equal $\{\perp\}$.

It remains to prove the universal property. Given a further candidate $S$, i.e. a large Lawvere theory with morphisms $G: L \rightarrow S$ and $G_{\mathcal{P}}: L_{\mathcal{P}} \rightarrow S$ satisfying the tensor law, we define $\bar{G}_{0}: T_{0} \rightarrow S$ as before; the proof that $\bar{G}_{0}$ respects composition and [_] is unchanged from the nonempty case. Again, it is clear that $\bar{G}_{0}$ factors through $T$ because its kernel satisfies the inductive definition of $\approx$, including all instances of $(\perp)$ as these are implied by validity of the tensor law in $S$. Uniqueness of the factorization is, again, clear.

\section{F. Proof of Lemma 30}

We need a preliminary lemma:

Lemma 41. Let $A: n \rightarrow m$ in $T_{0}$. Then for all $a: n \rightarrow m$ and all $b: m \rightarrow k$,

$$
a \in \mathrm{cl}(A) \Longrightarrow b a \in \operatorname{cl}(b A) .
$$

Proof: It suffices to show that the set $\bar{A}=\{a \in \operatorname{cl}(A) \mid$ $b a \in \operatorname{cl}(b A)\}$ contains $A$, is downward closed, and is closed under $(\Delta)$. The first and second properties are clear; we check the third property. Thus, let $h: n \rightarrow k$ and $g: k \rightarrow m$ such that $g \Delta_{j} h \in \bar{A}$ for all $j \in k$. Then $b g \Delta_{j} h \in \operatorname{cl}(b A)$ for all $j$, and hence $b g h \in \mathrm{cl}(b A)$, so that $g h \in \bar{A}$ as required.

Proof of Lemma 30: In preparation, note that

$$
\Delta_{i}=\left\langle\delta_{i j}\right\rangle_{j \in n}\left[\kappa_{i}\right]
$$

Only if: It suffices to show that the equivalence $\simeq$ defined by $A \simeq B$ iff $\mathrm{cl}(A)=\mathrm{cl}(B)$ is closed under $(\pi)$ and contains all instances of $(\perp)$. The latter holds by the definition of $\mathrm{cl}(\varnothing)$. To check the former, we first show that $\simeq$ is left congruent w.r.t. composition. Thus, let $A, B: n \rightarrow m$ and let $C: m \rightarrow k$ such that $\mathrm{cl}(A)=\mathrm{cl}(B)$. We have to show $\mathrm{cl}(C A)=\operatorname{cl}(C B)$. Since $\mathrm{cl}(C B)$ is downward closed and closed under $(\Delta)$, it suffices to prove $C A \subseteq \operatorname{cl}(C B)$. Thus let $c \in C, a \in A$. Then $a \in \operatorname{cl}(B)$ by assumption, and therefore $c a \in \mathrm{cl}(c B) \subseteq \mathrm{cl}(C B)$ by Lemma 41). It follows that $\mathrm{cl}(C A) \subseteq \mathrm{cl}(C B)$. The converse implication is shown symmetrically.

It remains to show that $\simeq$ is closed under $(\pi)$. Thus, let $A, B: n \rightarrow m$ such that $\mathrm{cl}\left(\left[\kappa_{i}\right] A\right)=\mathrm{cl}\left(\left[\kappa_{i}\right] B\right)$ for all $i \in m$. We have to show $\operatorname{cl}(A)=\operatorname{cl}(B)$. By (10) and Lemma 41, we have $\Delta_{i} a \in \mathrm{cl}\left(\left(\Delta_{i} B\right)\right)$ for all $i \in m, a \in A$. By downward closedness, $\mathrm{cl}\left(\left(\Delta_{i} B\right)\right) \subseteq \mathrm{cl}(B)$, so that we obtain $a \in \mathrm{cl}(B)$ by rule $(\Delta)$. It follows that $\mathrm{cl}(A) \subseteq \mathrm{cl}(B)$; the reverse inclusion is shown symmetrically.

If: It suffices to show that for $A: n \rightarrow m$ in $T_{0}, A \approx$ $\operatorname{cl}(A)$. Since $\approx$ is congruent w.r.t. set union, it suffices to show that $A \approx A \cup\{f\}$ for all $f \in \operatorname{cl}(A)$, which will follow if we show that the set

$$
\bar{A}:=\{f: n \rightarrow m \mid A \approx A \cup\{f\}\}
$$

(which clearly contains $A$ ) is downward closed and closed under $\Delta$.

- $\bar{A}$ is downward closed: define a preorder $\unlhd$ by $f \unlhd g$ : $\Longleftrightarrow\{f, g\} \approx g$; then $\unlhd$ is easily seen to be closed under $\pi_{\sqsubseteq}$, and hence contains $\sqsubseteq$. Now let $g \in \bar{A}, f \sqsubseteq g$. Then $f \unlhd g$ and therefore

$$
A \cup\{f\} \approx A \cup\{g\} \cup\{f\} \approx A \cup\{g\} \approx A,
$$

using congruence of $\approx$ w.r.t. union.

- $\bar{A}$ is closed under $(\Delta)$ : Let $f: n \rightarrow m, g: m \rightarrow k$, and let $f \Delta_{i} g \in \bar{A}$ for all $i \in m$. Then

$$
A \cup\{f g\} \approx A \cup\left\{f \Delta_{i} g \mid i \in m\right\} \approx A,
$$

using congruence w.r.t. union and Lemma 28.

G. Proof of Lemma 28

By rule $(\pi)$, it suffices to prove $\left[\kappa_{j}\right] g \approx\left[\kappa_{j}\right]\left\{\Delta_{i} g \mid i \in m\right\}$ for all $j \in m$. But the right hand side of this equivalence equals $\left\{\left[\kappa_{j}\right] g\right\} \cup\{\perp\}$, which is equivalent to $\left[\kappa_{j}\right] g$ by $(\perp)$ and congruence w.r.t. union. 
H. Proof of the Order-Theoretic Conservativity Theorem 32

1) a) $\Rightarrow b$ ): Immediate from Lemma 28 .

b) $\Rightarrow c)$ : The inclusion $\mathrm{cl}(f) \subseteq f \downarrow$ holds because under b), $f \downarrow$ is closed under $(\Delta)$. The reverse inclusion holds because $\mathrm{cl}(f)$ is downclosed.

c) $\Rightarrow a$ ): Immediate by Lemma 30 .

2) Immediate from property c).

\section{Details for Remark 34}

Let $L$ be simply ordered. We prove that if $\sigma_{1}: L \rightarrow L \otimes$ $L_{\mathcal{P}}$ is faithful, then it reflects the ordering. According to Theorem 32, we have to show that for $f: n \rightarrow m, g$ : $m \rightarrow k$ in $L, f g=\bigsqcup_{i \in m} f \Delta_{i} g$. Thus let $h$ be an upper bound of $\left\{f \Delta_{i} g \mid i \in m\right\}$. Since $L$ is simply ordered, there exist $f^{\prime}, g^{\prime}$ such that $f^{\prime} g^{\prime} \sqsubseteq h, f^{\prime} g^{\prime}$ is a minimal upper bound of $\left\{f \Delta_{i} g \mid i \in m\right\}$, and for every $j$ there is $i$ such that $f^{\prime} \Delta_{j} g^{\prime} \sqsubseteq f \Delta_{i} g \sqsubseteq f g$. By $(\Delta), f^{\prime} g^{\prime} \in \mathrm{cl}(f g)$ and $f g \in \mathrm{cl}\left(f^{\prime} g^{\prime}\right)$. Hence $\mathrm{cl}(f g)=\mathrm{cl}\left(f^{\prime} g^{\prime}\right)$. Faithfulness of $\sigma_{1}$ then implies that $f g=f^{\prime} g^{\prime} \sqsubseteq h$.

\section{J. Details for Example 35}

Absolutely free theories: For the sake of readability, we restrict to the case where $L$ is generated by a finitary signature $\Sigma$ (and, of course, no equations). Then $L \otimes L_{\perp}$ is generated by $\Sigma$ and an additional constant $\perp$, and equations

$$
f(\perp, \ldots, \perp)=\perp
$$

for every basic function symbol $f$ in $\Sigma$. We can direct these equations from left to right and obtain a single-step rewrite relation $\rightarrow$ which is clearly strongly normalizing and trivially locally confluent, hence confluent by Newman's lemma. We define a syntactic approximation $\sqsubseteq_{0}$ on $\Sigma \cup\{\perp\}$ terms by $t \sqsubseteq_{0} s$ iff there exist variables $x_{1}, \ldots, x_{n}$ such that $t=s\left[\perp / x_{1}, \ldots, \perp / x_{n}\right]$; i.e. $t \sqsubseteq_{0} s$ iff the term $t$ is obtained from $s$ by deleting some subterms. Then

Claim 42. $t \sqsubseteq s$ iff $N F(t) \sqsubseteq_{0} s$

where $N F(t)$ denotes the normal form w.r.t. $\rightarrow$. 'If' is trivial since $t \sqsubseteq 0 s$ clearly implies $t \sqsubseteq s$.

To prove 'only if', first note that $t \sqsubseteq s$ iff $s$ can be reached from $t$ by a chain of terms $t=t_{0}, \ldots, t_{n}=s$ such that for each $i=0, \ldots, n-1$, either $t_{i} \sqsubseteq_{0} t_{i+1}$ or $t_{i}=t_{i+1}$ modulo the equations. We now show that whenever $t \sqsubseteq_{0} s \leftrightarrow s^{\prime}$, then there exists $t^{\prime}$ such that $t \rightarrow^{*} t^{\prime} \sqsubseteq_{0} s^{\prime}$, where $\leftrightarrow$ denotes the symmetric closure of $\rightarrow$, and $\rightarrow^{*}$ the transitive reflexive closure. Since $s^{\prime} \rightarrow s$ implies $s \sqsubseteq_{0} s^{\prime}$ and $\sqsubseteq_{0}$ is clearly a partial order, it suffices to consider the case $s \rightarrow s^{\prime}$, that is, $s^{\prime}$ is obtained from $s$ by deleting an occurrence $r$ of a subterm of the form $f(\perp, \ldots, \perp)$. If $r$ is still present in $t$, we can delete it in $t$, obtaining $t^{\prime}$ such that $t \rightarrow t^{\prime}$; then clearly $t^{\prime} \sqsubseteq_{0} s^{\prime}$. If $r$ is deleted in $t$, then already $t \sqsubseteq_{0} s^{\prime}$.

Now it follows that $t \sqsubseteq s$ iff there exists $t^{\prime}$ such that $t \rightarrow^{*}$ $t^{\prime} \sqsubseteq s$. We are done by noting that $N F(t)=N F\left(t^{\prime}\right) \sqsubseteq_{0} t^{\prime}$. This proves Claim 42.
We now set out to prove the equality $\bigsqcup_{i \in m} f \Delta_{i} g=f g$ for $g: n \rightarrow m, f: m \rightarrow k$. Translating this into the language of $\Sigma \cup\{\perp\}$-terms, we have to show for a term $t$ in variables $x, \ldots, x_{m}$ and terms $s_{i}$ that $\bigsqcup t \sigma_{i}=t \sigma$, where the substitutions $\sigma, \sigma_{i}$ are defined by $\sigma\left(x_{i}\right)=s_{i}$ and by $\sigma_{i}\left(x_{i}\right)=s_{i}, \sigma_{i}\left(x_{j}\right)=\perp$ for $i \neq j$, respectively. Thus, $t \sigma_{i} \sqsubseteq_{0} t \sigma$ for all $i$, i.e. $t \sigma$ is an upper bound. Let $h$ be a further upper bound of the $t \sigma_{i}$ w.r.t. $\sqsubseteq$. By Claim 42, $N F\left(t \sigma_{i}\right) \sqsubseteq_{0} h$ for all $i$. We are done once we prove that $N F(t \sigma)$ is the least upper bound of the $N F\left(t \sigma_{i}\right)$ w.r.t. $\sqsubseteq_{0}$. This just means that a subterm at position $r$ in $t \sigma$ is deleted in $N F(t \sigma)$ iff it is deleted in all of the $t \sigma_{i}$. Here, 'only if' is clear (since the $\sigma_{i}$ only introduce more occurrences of $\perp)$. To prove 'if', we proceed as follows. If $r$ lies within a substituted occurrence of $x_{i}$, i.e. within an occurrence of $s_{i}$, then the subterm at position $r$ is the same in $t \sigma$ as in $t \sigma_{i}$, and we are done. Otherwise, the position $r$ lies within $t$. Our goal then reduces to showing that for every term $q$ in the variables $x_{i}, q \sigma_{i}=\perp$ (modulo the equations) for all $i$ implies $q \sigma=\perp$. We prove this by induction on the term structure. The base case (where $q$ is one of the variables $x_{i}$ ) is clear. If $q$ is of the form $f\left(p_{1}, \ldots, p_{w}\right)$, then $q \sigma_{i}=\perp$ implies (by confluence of $\rightarrow$ ) that $p_{j} \sigma_{i}=\perp$ for all $i, j$, so that $p_{j} \sigma=\perp$ for all $j$ by induction, and hence $q \sigma=\perp$.

Finitely additive finitary Lawvere theories: Let $L$ be a finitely additive finitary Lawvere theory; we prove that $L$ admits unbounded nondeterminism. Recall that by Lemma 24, the approximation ordering on $L$ coincides with the ordering induced by the additive structure. By Theorem 32, all that remains to be shown is that for all $f: n \rightarrow m, g: m \rightarrow k$ in $L, f g=\sum_{j \in m} f \Delta_{j} g$, where we can restrict to finite $n, m, k$ because $L$ is finitary. Since finite sums commute with composition, this reduces to showing that $\sum_{j \in m} \Delta_{j}=\mathrm{id}_{m}$, which is straightforward by comparing projections. 